\title{
Pro Drupal 7 Development
}

Third Edition

\section{Todd Tomlinson \\ John K. VanDyk}




\title{
Pro Drupal 7 Development: Third Edition
}

\author{
Copyright (C) 2010 by Todd Tomlinson and John K. VanDyk
}

All rights reserved. No part of this work may be reproduced or transmitted in any form or by any means, electronic or mechanical, including photocopying, recording, or by any information storage or retrieval system, without the prior written permission of the copyright owner and the publisher.

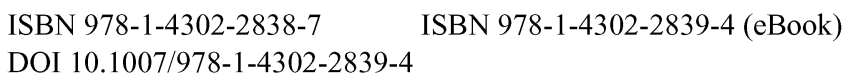

\section{1}

Trademarked names, logos, and images may appear in this book. Rather than use a trademark symbol with every occurrence of a trademarked name, logo, or image we use the names, logos, and images only in an editorial fashion and to the benefit of the trademark owner, with no intention of infringement of the trademark.

The use in this publication of trade names, trademarks, service marks, and similar terms, even if they are not identified as such, is not to be taken as an expression of opinion as to whether or not they are subject to proprietary rights.

\author{
President and Publisher: Paul Manning \\ Lead Editor: Michelle Lowman \\ Coordinating Editor: Anita Castro \\ Copy Editor: Mary Ann Fugate \\ Production Support: Patrick Cunningham \\ Indexer: BIM Indexing \& Proofreading Services \\ Artist: April Milne \\ Cover Designer: Anna Ishchenko
}

Technical Reviewers: Joshua Brauer, Robert Douglass, Peter M. Wolanin

Editorial Board: Steve Anglin, Mark Beckner, Ewan Buckingham, Gary Cornell, Jonathan Gennick, Jonathan Hassell, Michelle Lowman, Matthew Moodie, Duncan Parkes, Jeffrey Pepper, Frank Pohlmann, Douglas Pundick, Ben Renow-Clarke, Dominic Shakeshaft, Matt Wade, Tom Welsh

Distributed to the book trade worldwide by Springer Science+Business Media, LLC., 233 Spring Street, 6th Floor, New York, NY 10013. Phone 1-800-SPRINGER, fax (201) 348-4505, e-mail orders-ny@springer-sbm.com, or visit www.springeronline.com.

For information on translations, please e-mail rights@apress.com, or visitwww.apress.com.

Apress and friends of ED books may be purchased in bulk for academic, corporate, or promotional use. eBook versions and licenses are also available for most titles. For more information, reference our Special Bulk Sales-eBook Licensing web page at www. apress.com/info/bulksales.

The information in this book is distributed on an "as is" basis, without warranty. Although every precaution has been taken in the preparation of this work, neither the author(s) nor Apress shall have any liability to any person or entity with respect to any loss or damage caused or alleged to be caused directly or indirectly by the information contained in this work. 


\section{Contents at a Glance}

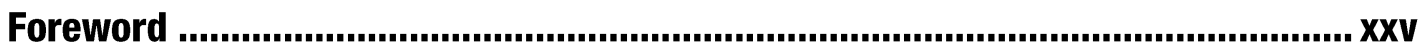

About the Authors................................................................................... Xxvi

About the Technical Reviewers ................................................................ xxvii

Acknowledgments ....................................................................................... Xxvifi

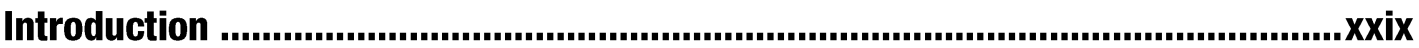

Chapter 1: How Drupal Works .....................................................................1

Chapter 2: Writing a Module .............................................................................13

Chapter 3: Hooks, Actions, and Triggers .............................................................. 33

Chapter 4: The Menu System...................................................................................57

Chapter 5: Working with Databases ...................................................................89

Chapter 6: Working with Users.......................................................................115

Chapter 7: Working with Nodes..................................................................137

Chapter 8: Working with Fields ...................................................................163

Chapter 9: The Theme System ..............................................................................185

Chapter 10: Working with Blocks ..............................................................223

Chapter 11: The Form API..............................................................................2239

Chapter 12: Manipulating User Input: The Filter System.................................295

Chapter 13: Searching and Indexing Content .................................................. 307

Chapter 14: Working with Files ............................................................................. 323 
Chapter 15: Working with Taxonomy ........................................................343

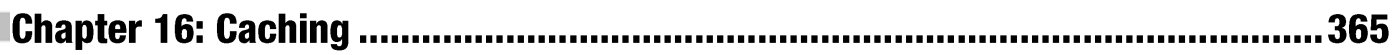

Chapter 17: Sessions ........................................................................... 379

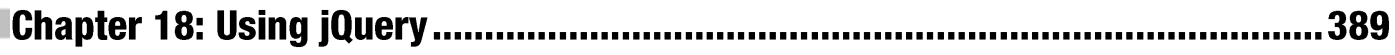

Chapter 10: Localization and Translation .....................................................417

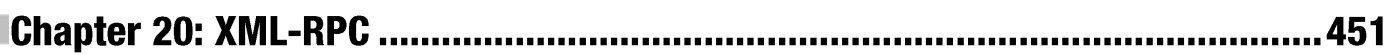

Chapter 21: Writing Secure Code...................................................................... 465

Chapter 22: Development Best Practices .................................................................487

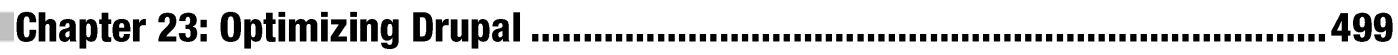

Chapter 24: Installation Profiles...........................................................525

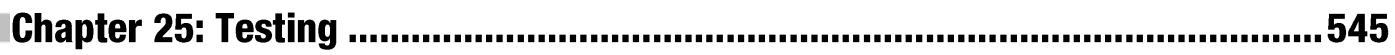

Appendix A: Database Table Reference ..........................................................565

Appendix B: Resources.............................................................................623

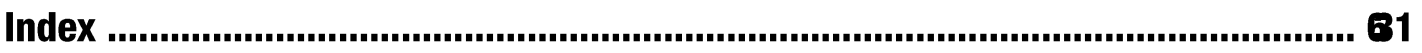




\section{Contents}

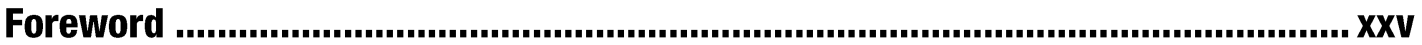

About the Authors................................................................................... Xxvi

About the Technical Reviewers .................................................................... xxvii

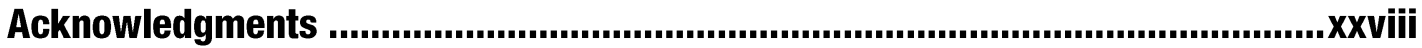

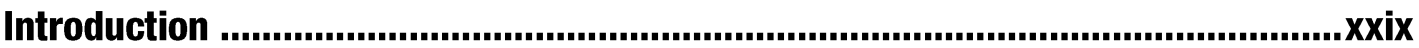

Chapter 1: How Drupal Works ....................................................................... 1

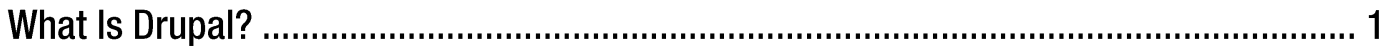

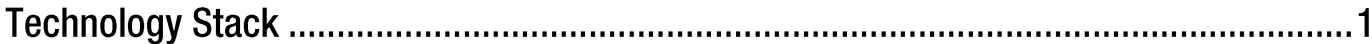

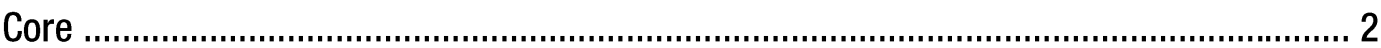

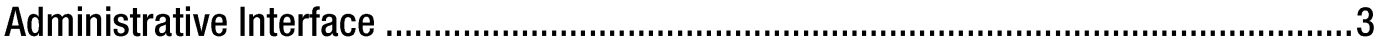

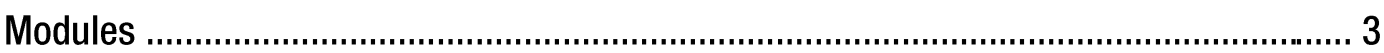

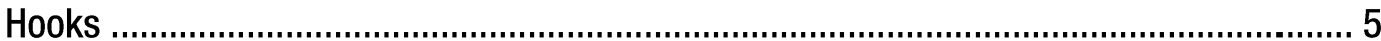

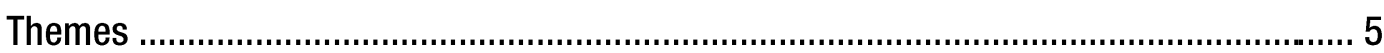

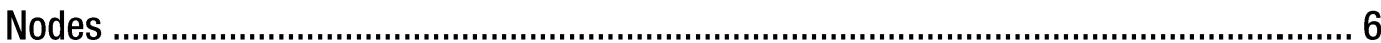

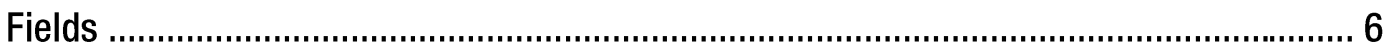

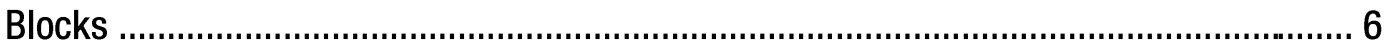

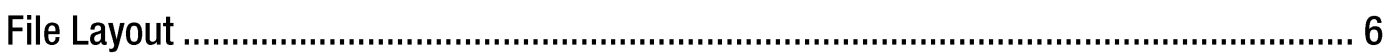

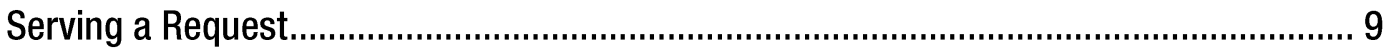

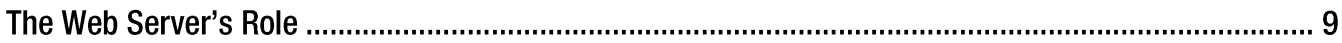

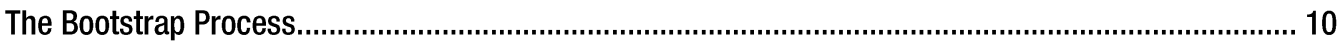




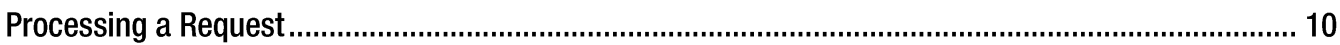

Theming the Data ......................................................................................................... 11

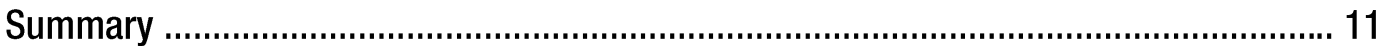

Chapter 2: Writing a Module .....................................................................13

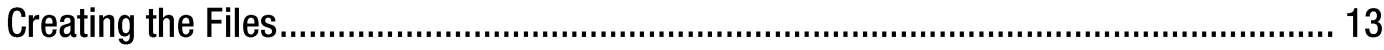

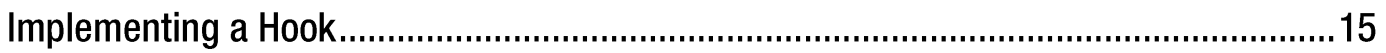

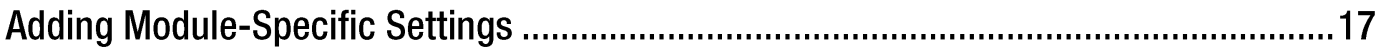

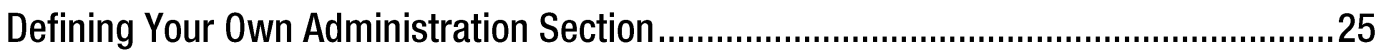

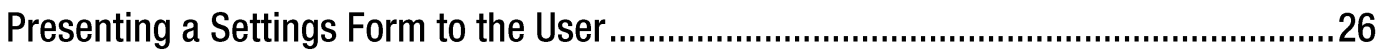

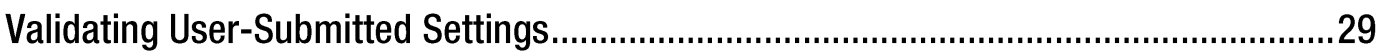

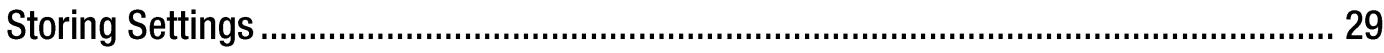

Using Drupal's variables Table ........................................................................................... 29

Retrieving Stored Values with variable_get() ............................................................................ 30

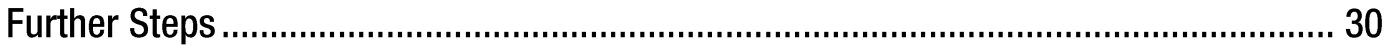

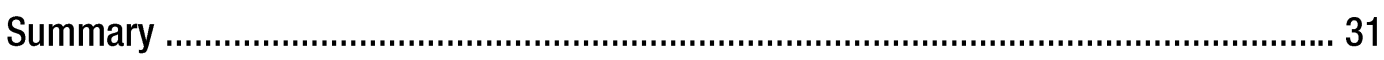

Chapter 3: Hooks, Actions, and Triggers .....................................................33

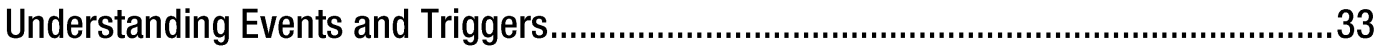

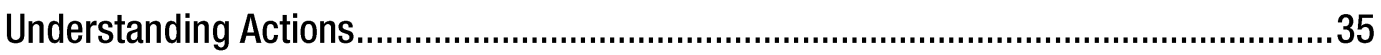

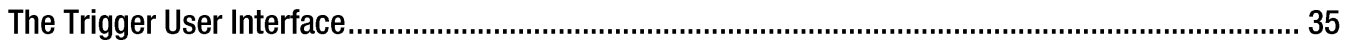

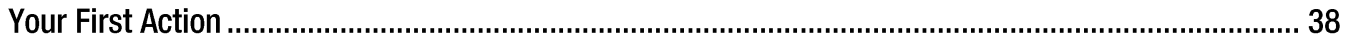

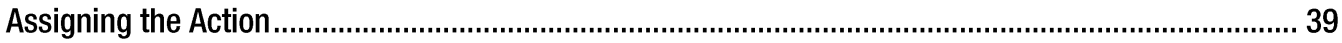

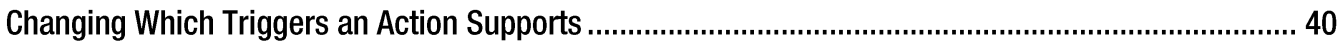

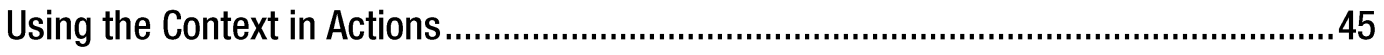

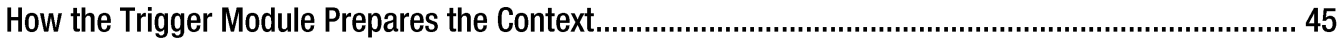

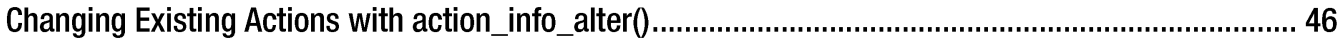

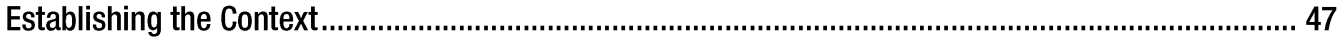




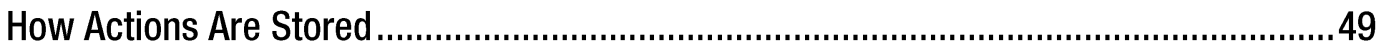

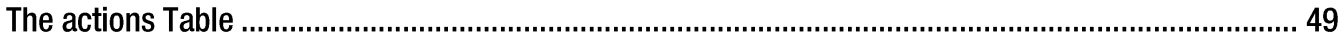

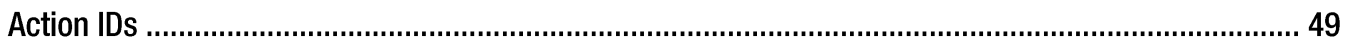

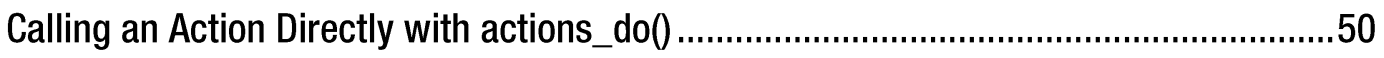

Defining Your Own Triggers with hook_trigger_info() ...............................................51

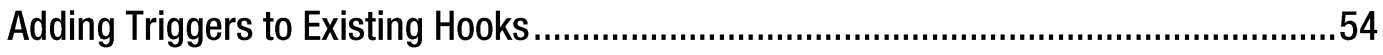

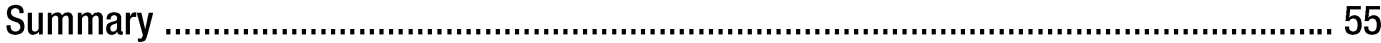

Chapter 4: The Menu System.......................................................................57

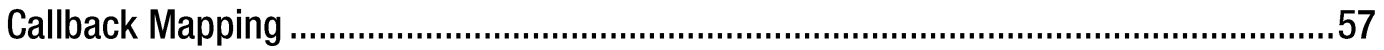

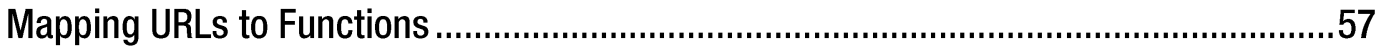

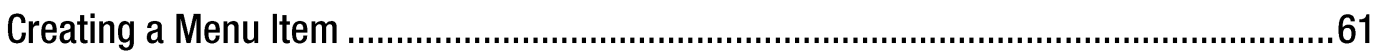

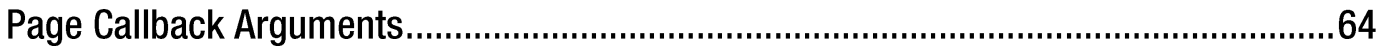

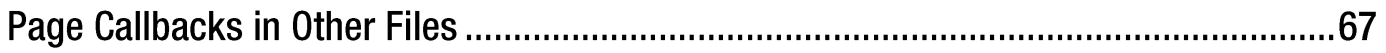

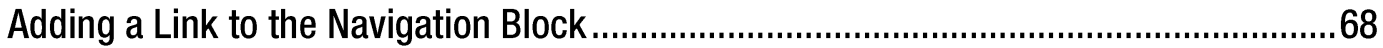

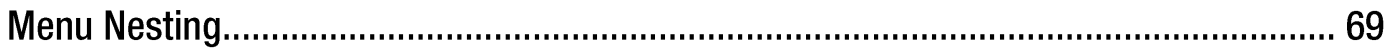

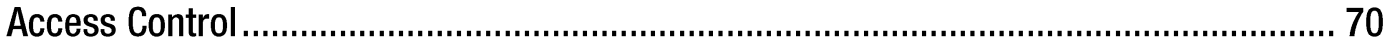

Title Localization and Customization .......................................................................

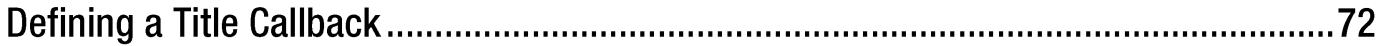

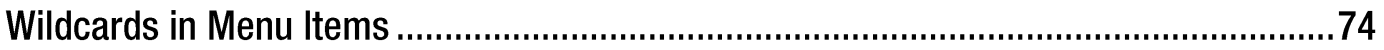

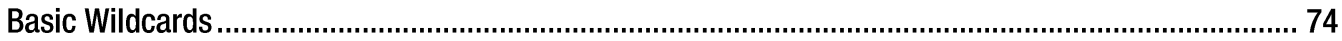

Wildcards and Page Callback Parameters.................................................................................. 75

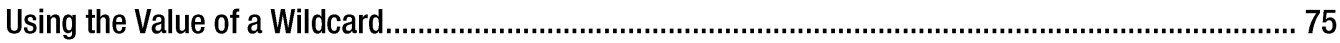

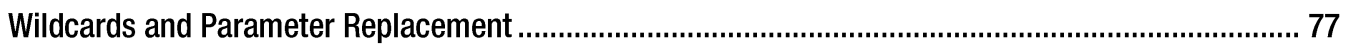

Passing Additional Arguments to the Load Function................................................................... 78

Special, Predefined Load Arguments: \%map and \%index ............................................................. 79

Building Paths from Wildcards Using to_arg() Functions ............................................................... 79

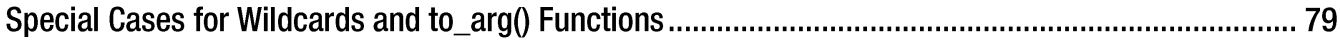




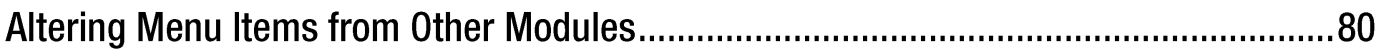

Altering Menu Links from Other Modules .................................................................... 82

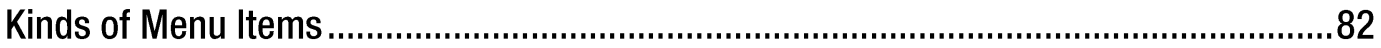

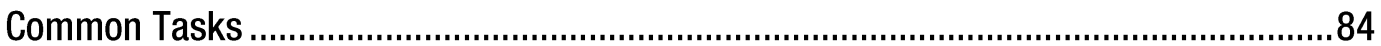

Assigning Callbacks Without Adding a Link to the Menu .............................................................. 85

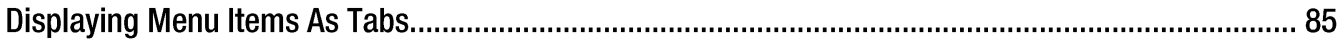

Hiding Existing Menu Items .............................................................................................. 87

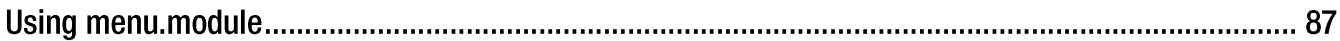

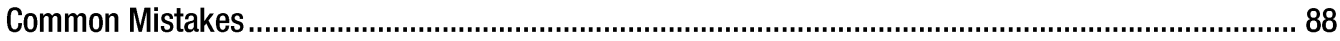

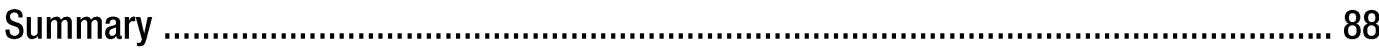

Chapter 5: Working with Databases ..............................................................89

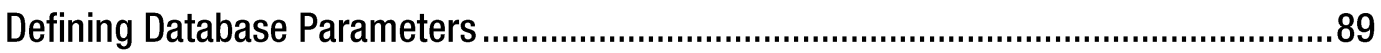

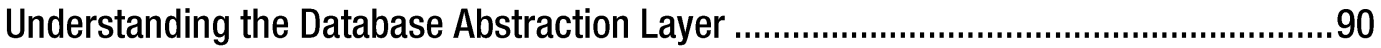

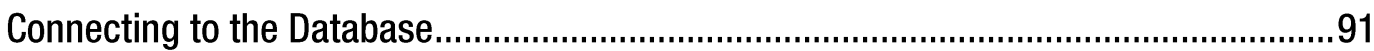

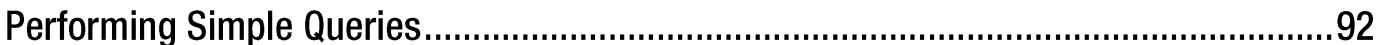

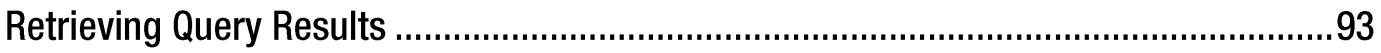

Getting a Single Value ………………….......................................................................... 94

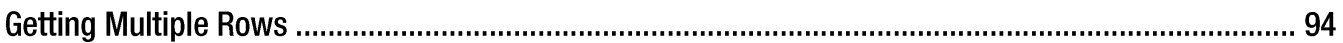

Using the Query Builder and Query Objects...................................................................................... 94

Getting a Limited Range of Results ...................................................................................... 95

Getting Results for Paged Display ......................................................................................... 96

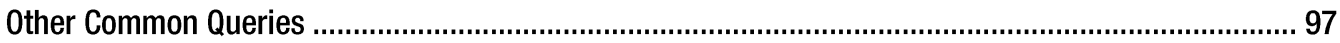

Inserts and Updates with drupal_write_record() .....................................................98

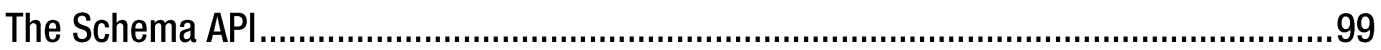

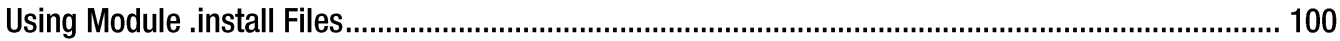

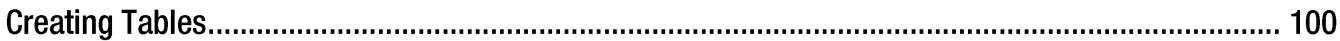

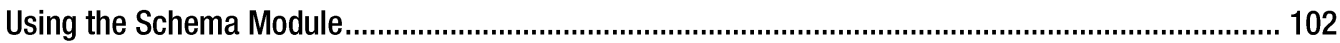

Field Type Mapping from Schema to Database.............................................................................. 103 
Declaring a Specific Column Type with mysql_type ..................................................................... 106

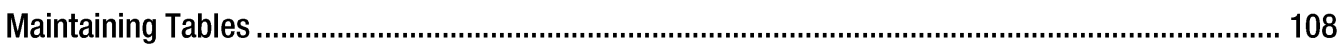

Deleting Tables on Uninstall .................................................................................................. 109

Changing Existing Schemas with hook_schema_alter( …….................................................... 110

Modifying Other Modules' Queries with hook_query_alter() ........................................111

Connecting to Multiple Databases Within Drupal ......................................................112

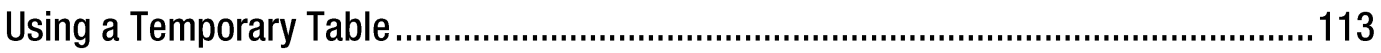

Writing Your Own Database Driver ............................................................................114

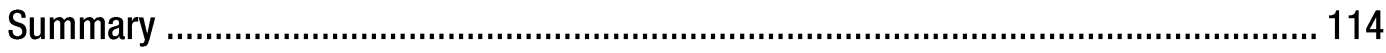

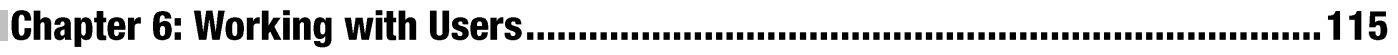

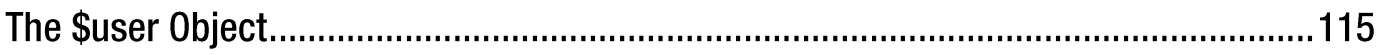

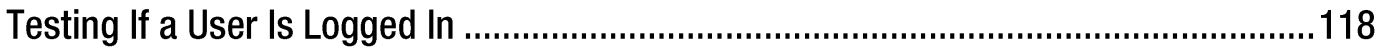

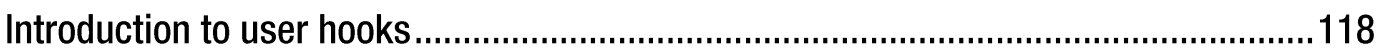

Understanding hook_user_view(\$account, \$view_mode) ……………......................................... 120

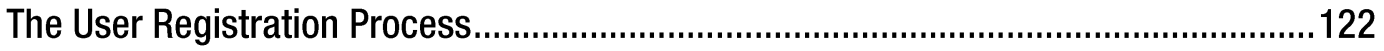

Using profile.module to Collect User Information .....................................................125

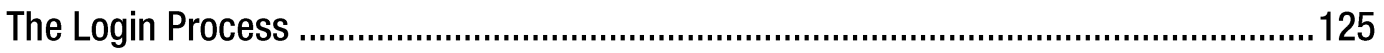

Adding Data to the \$user Object at Load Time ....................................................................... 127

Providing User Information Categories.................................................................................... 129

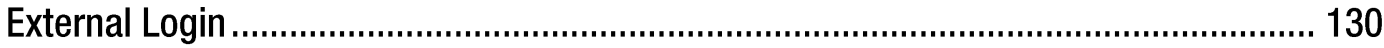

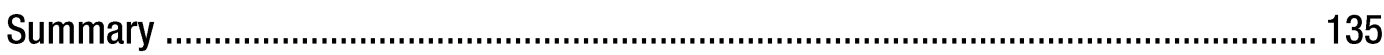

Chapter 7: Working with Nodes.........................................................................137

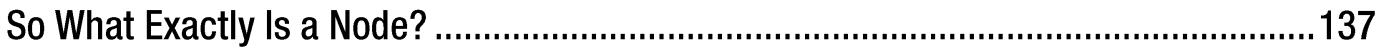

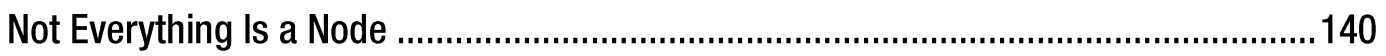

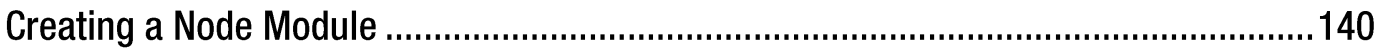

Creating the install File................................................................................................... 140

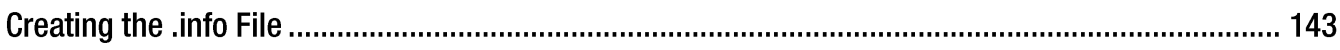




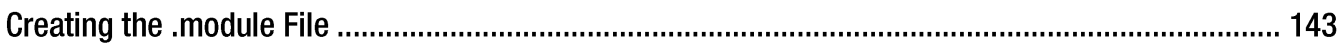

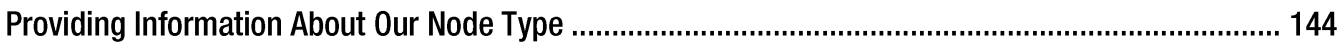

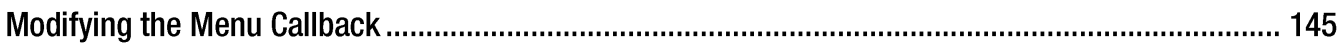

Defining Node-Type-Specific Permissions with hook_permission() .............................................. 146

Limiting Access to a Node Type with hook_node_access() .......................................................... 147

Customizing the Node Form for Our Node Type ....................................................................... 148

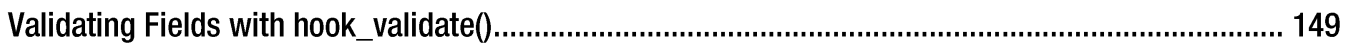

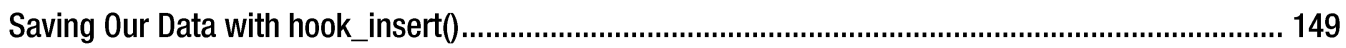

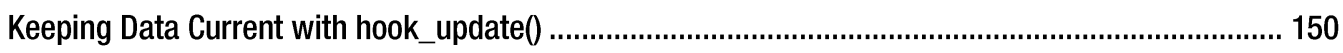

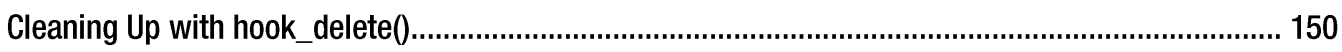

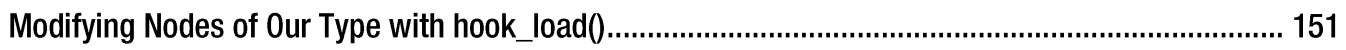

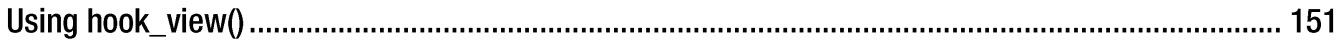

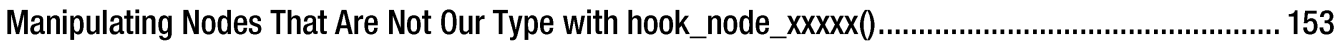

How Nodes Are Stored.................................................................................... 155

Creating a Node Type with Custom Content Types.................................................... 157

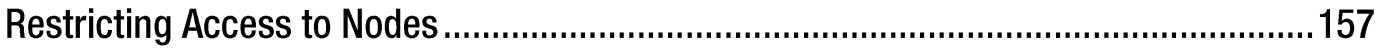

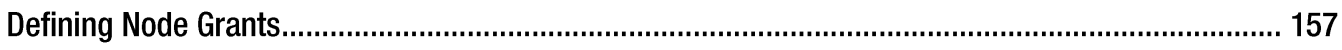

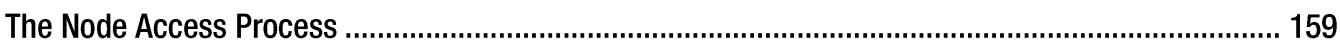

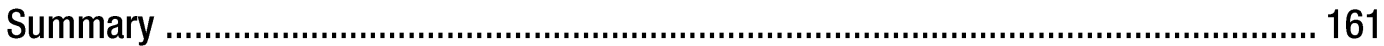

Chapter 8: Working with Fields ......................................................... 163

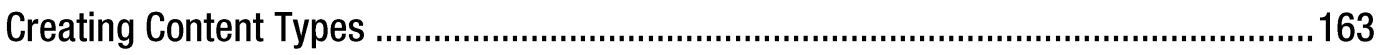

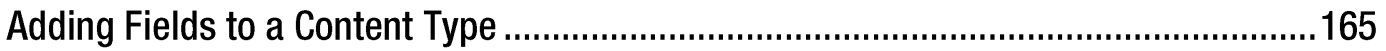

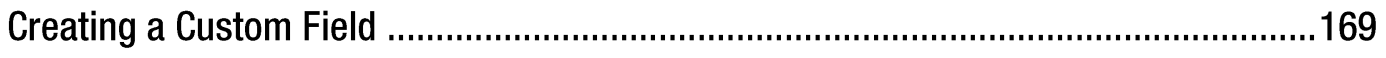

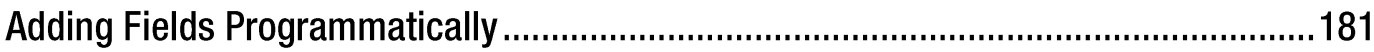

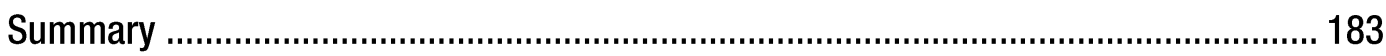


Chapter 9: The Theme System ................................................................185

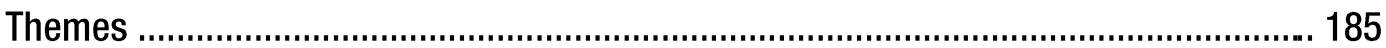

Installing an Off-the-Shelf Theme ………........................................................................ 185

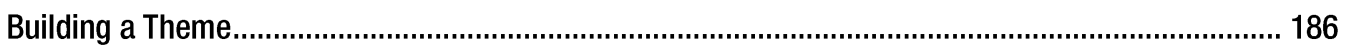

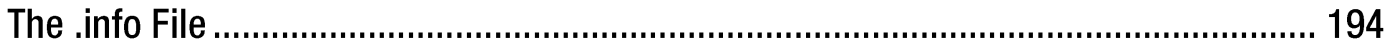

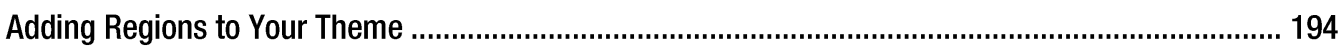

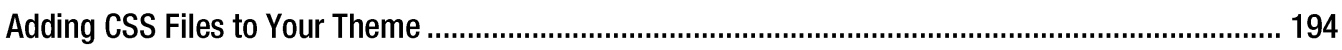

Adding JavaScript Files ..................................................................................................... 195

Adding Settings to Your Theme ......................................................................................... 195

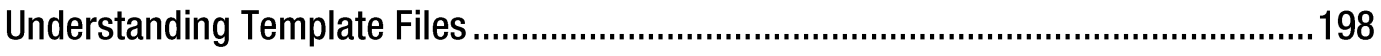

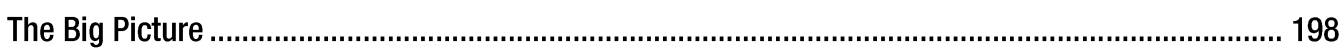

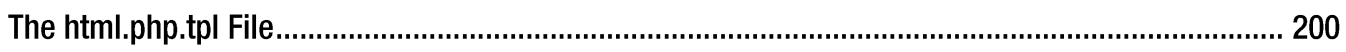

Overriding Template Files................................................................................................. 214

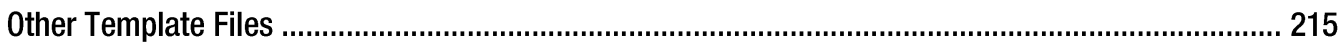

Overriding Themable Items ................................................................................................. 216

Overriding with Template Files......................................................................................... 219

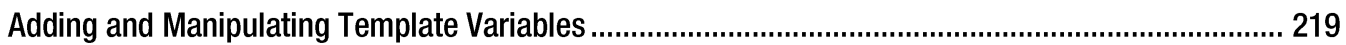

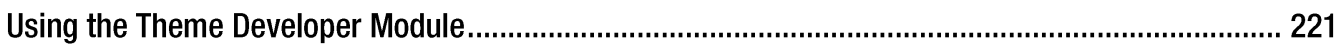

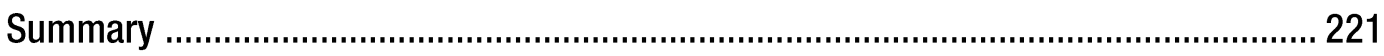

Chapter 10: Working with Blocks...............................................................223

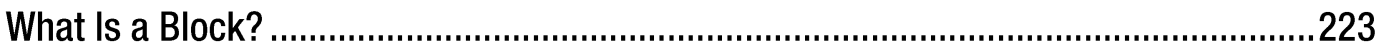

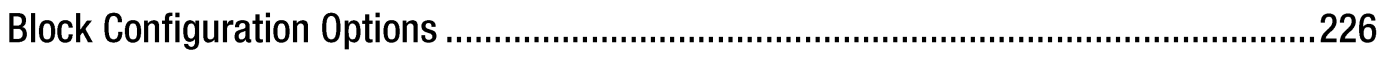

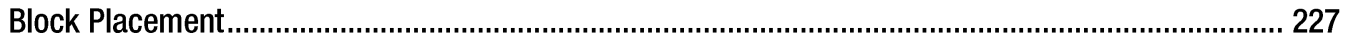

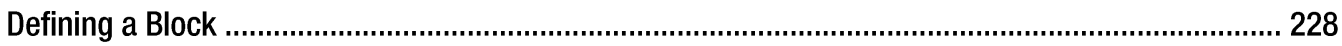

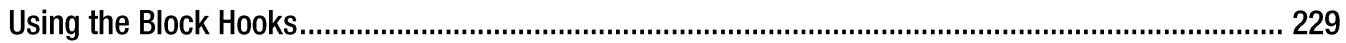

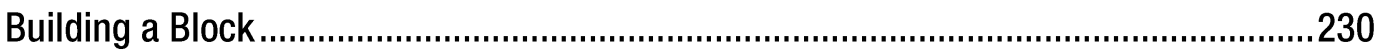

Enabling a Block When a Module Is Installed ...........................................................2. 


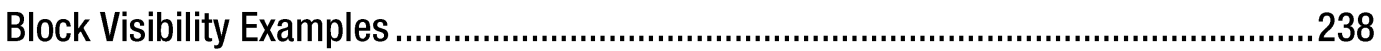

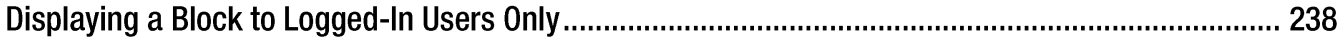

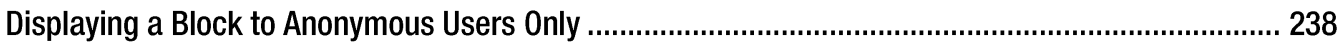

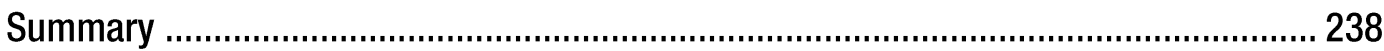

Chapter 11: The Form API.............................................................................239

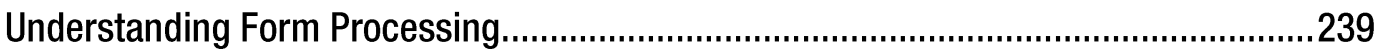

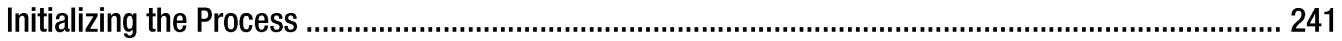

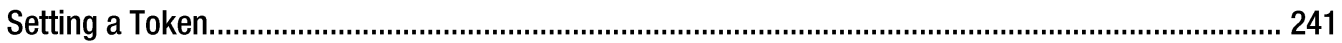

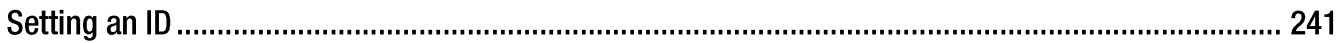

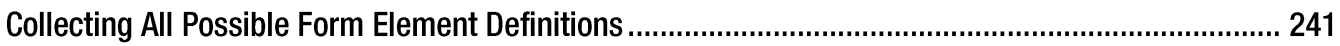

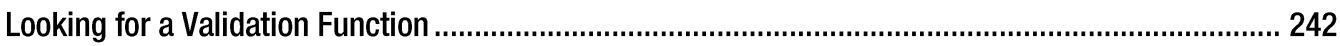

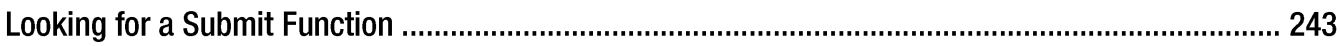

Allowing Modules to Alter the Form Before It's Built....................................................................... 243

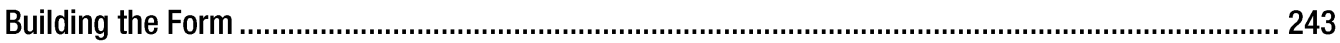

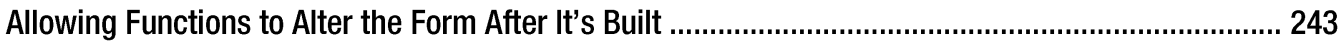

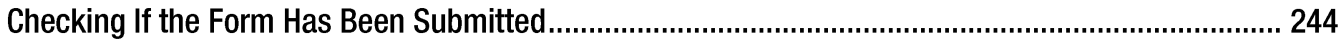

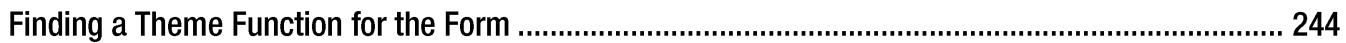

Allowing Modules to Modify the Form Before It's Rendered ......................................................... 244

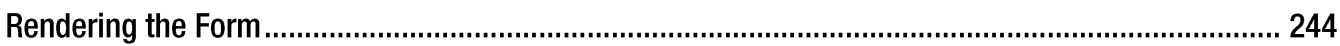

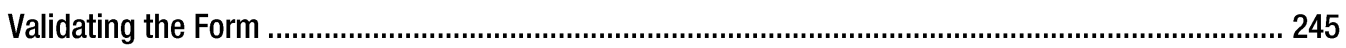

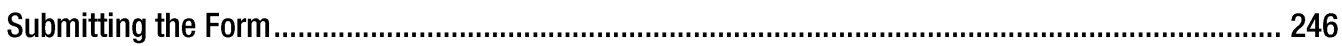

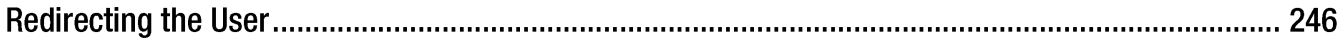

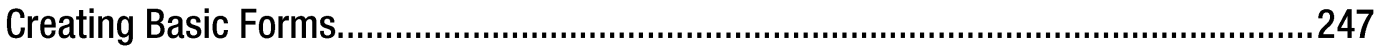

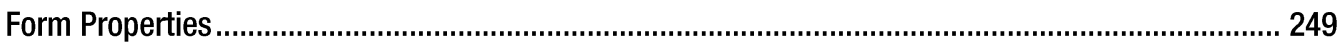

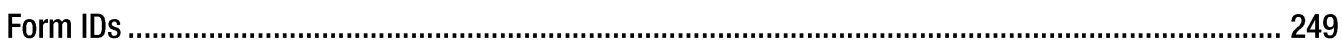

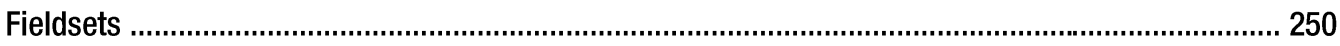

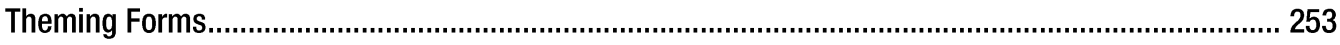

Specifying Validation and Submission Functions with hook_forms()_........................................... 257

Call Order of Theme, Validation, and Submission Functions ......................................................... 258 


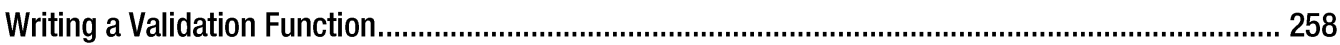

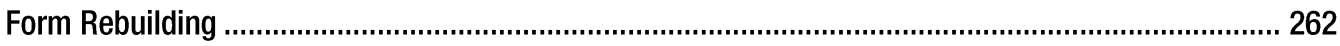

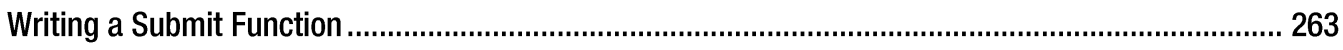

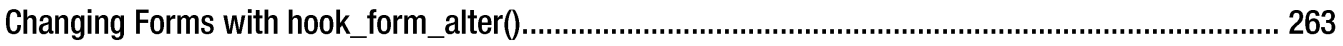

Submitting Forms Programmatically with drupal_form_submit() .................................................. 265

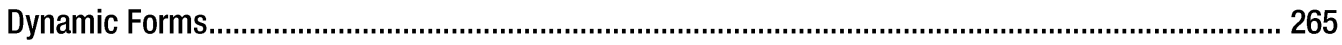

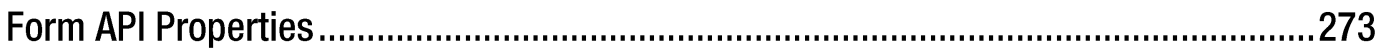

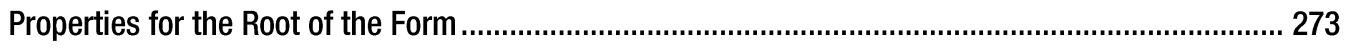

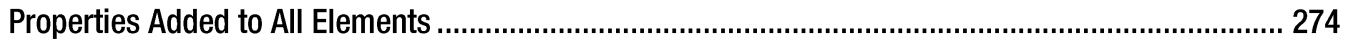

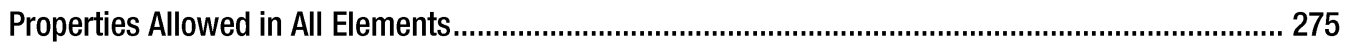

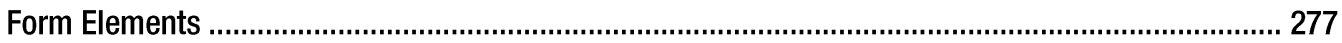

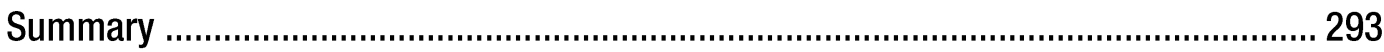

Chapter 12: Manipulating User Input: The Filter System.............................295

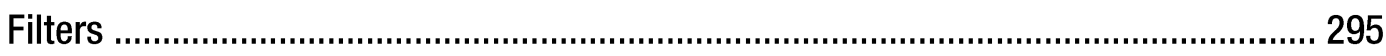

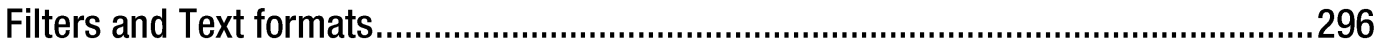

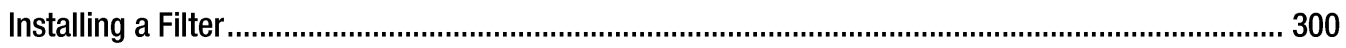

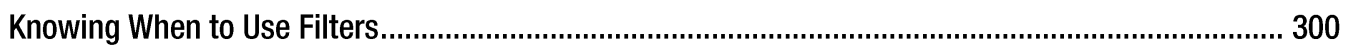

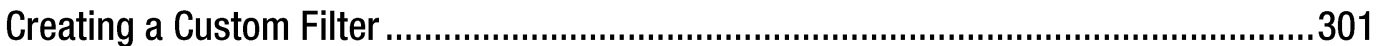

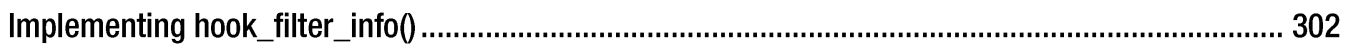

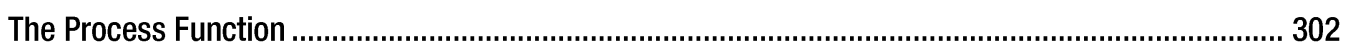

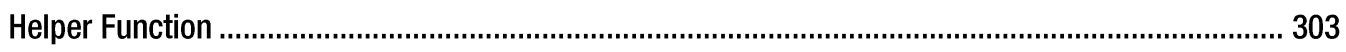

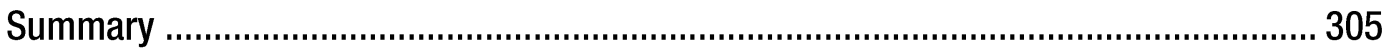

Chapter 13: Searching and Indexing Content ............................................. 307

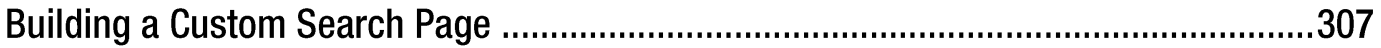

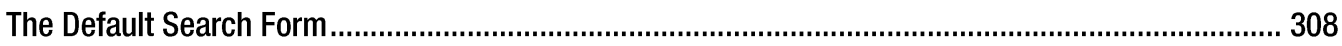

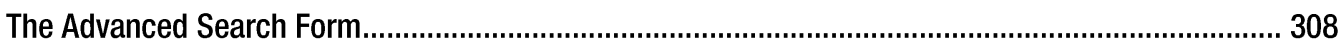

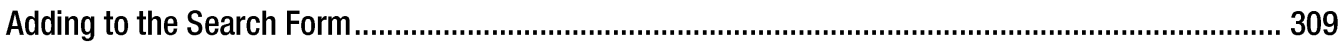




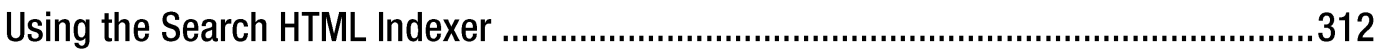

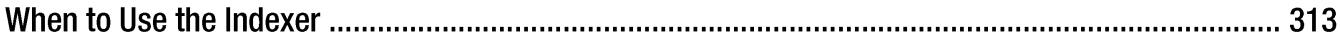

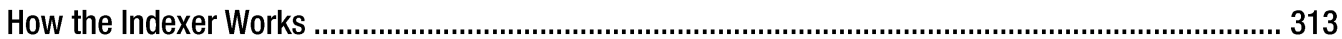

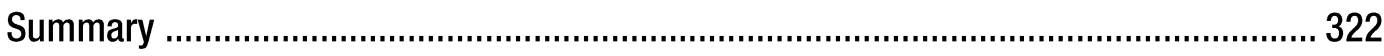

Chapter 14: Working with Files ....................................................................... 323

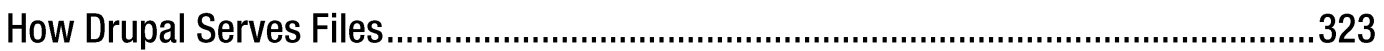

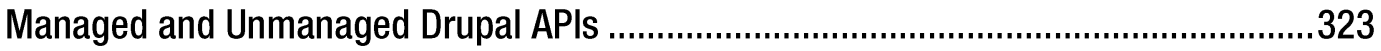

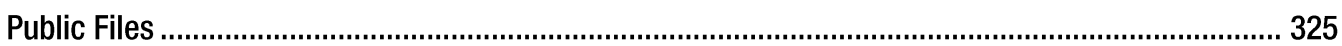

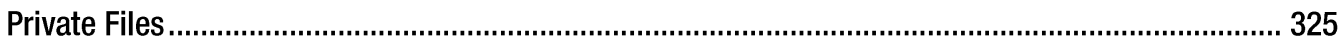

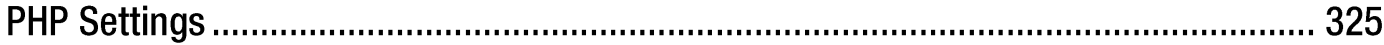

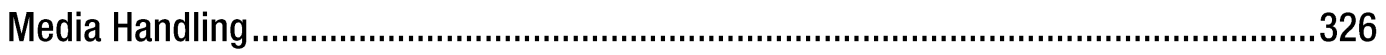

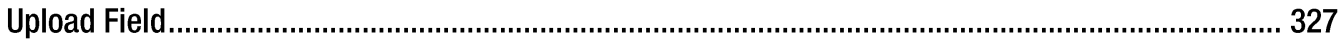

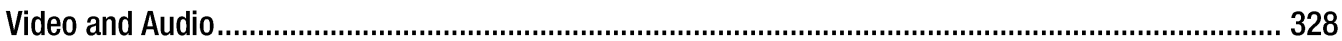

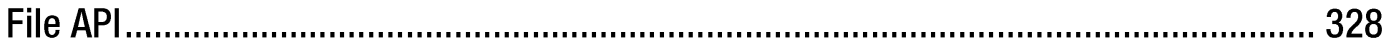

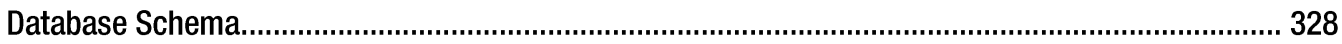

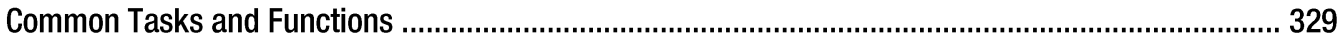

Authentication Hooks for Downloading ................................................................................... 340

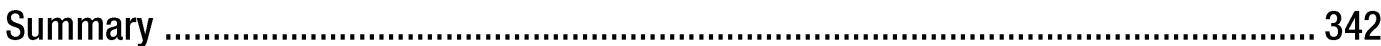

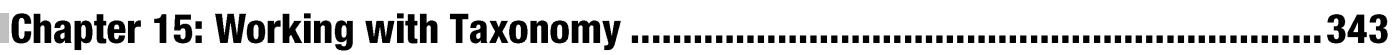

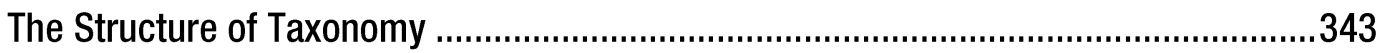

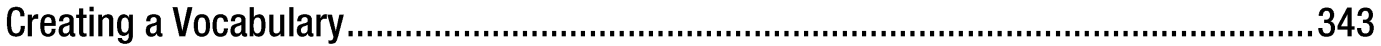

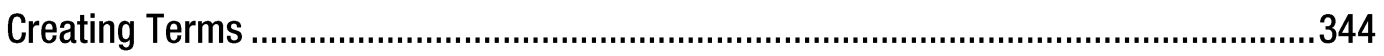

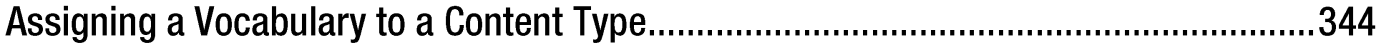

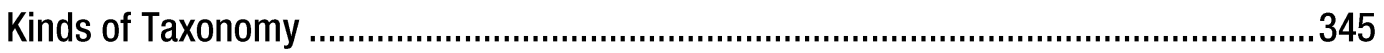

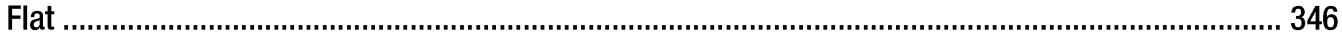

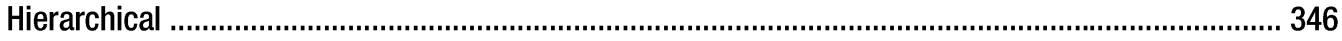

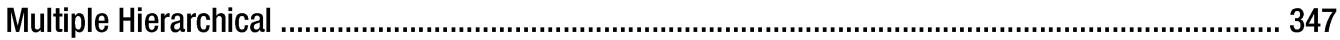




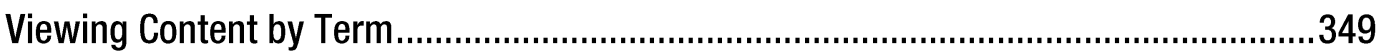

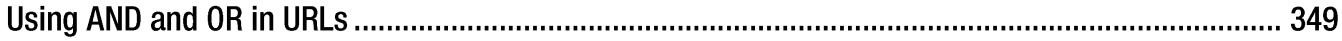

Specifying Depth for Hierarchical Vocabularies .......................................................................... 349

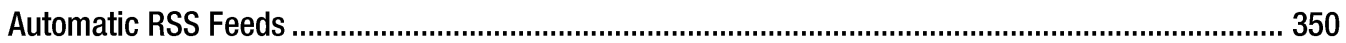

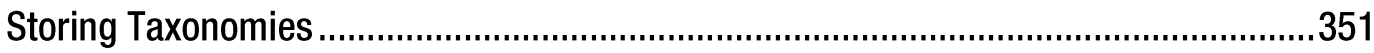

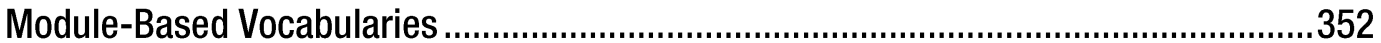

Creating a Module-Based Vocabulary ...................................................................................... 352

Keeping Informed of Vocabulary Changes with Taxonomy Hooks ................................................ 352

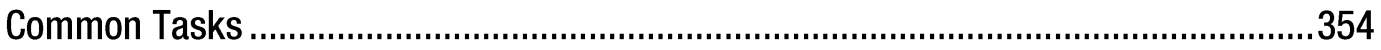

Displaying Taxonomy Terms Associated with a Node …………...................................................... 354

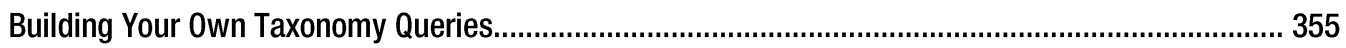

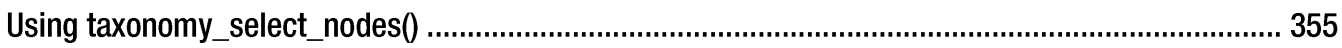

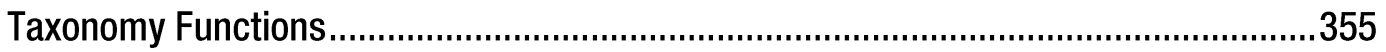

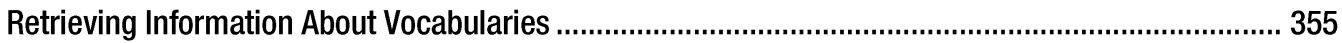

Adding, Modifying, and Deleting Vocabularies .......................................................................... 356

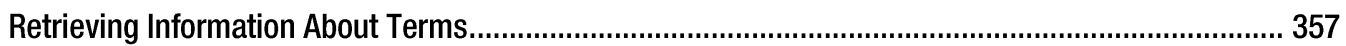

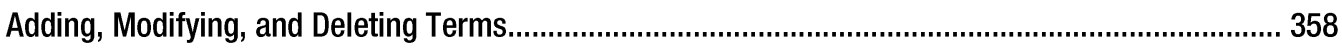

Retrieving Information About Term Hierarchy ........................................................................... 359

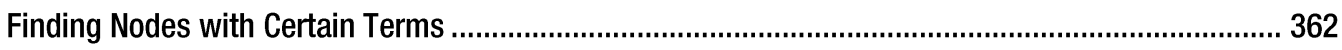

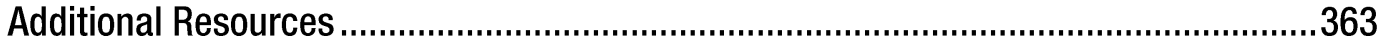

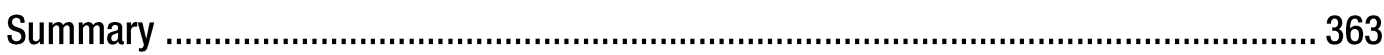

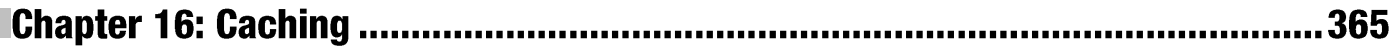

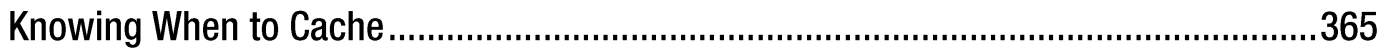

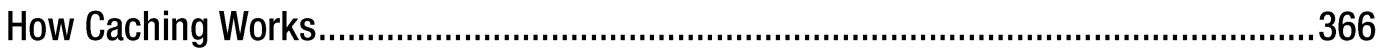

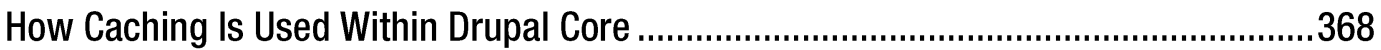

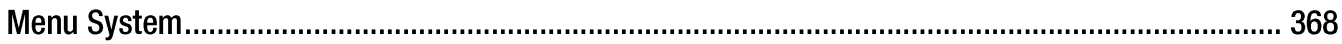

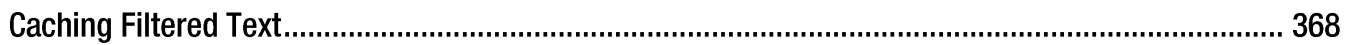

Administration Variables and Module Settings.......................................................................... 369 


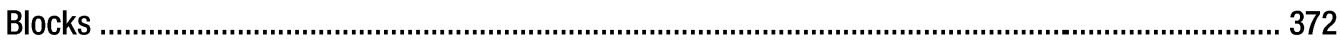

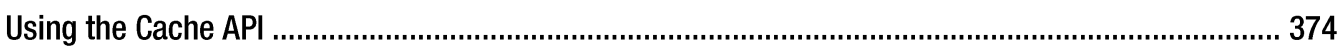

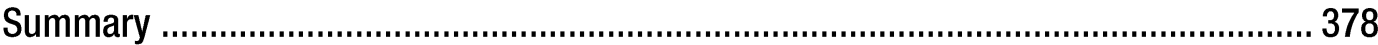

Chapter 17: Sessions .............................................................................379

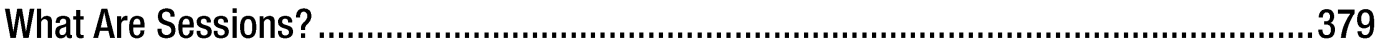

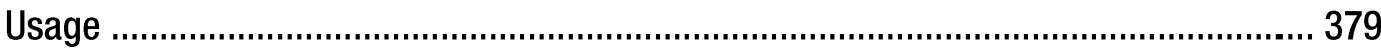

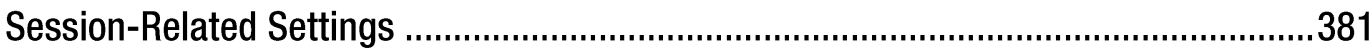

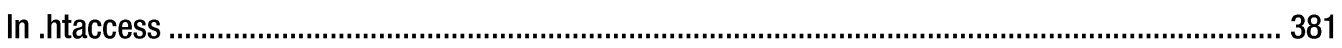

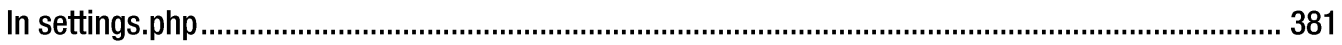

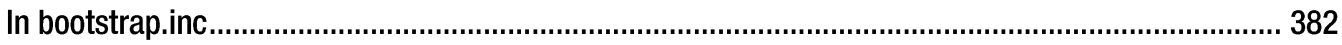

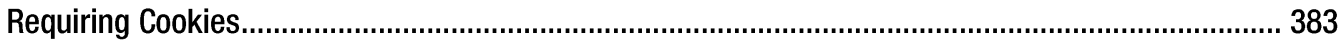

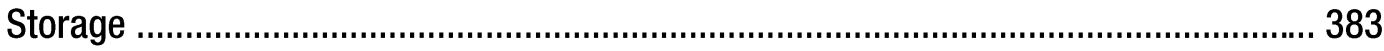

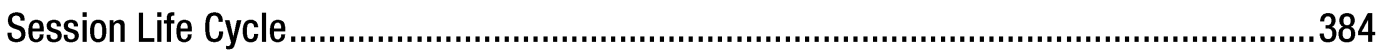

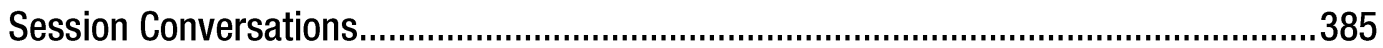

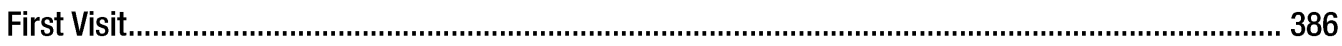

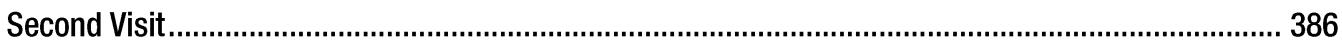

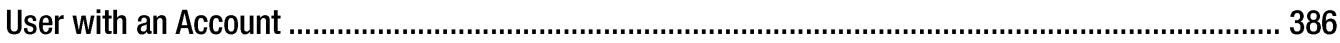

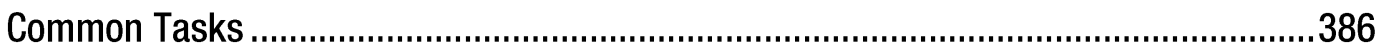

Changing the Length of Time Before a Cookie Expires..................................................................... 386

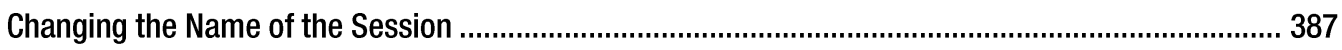

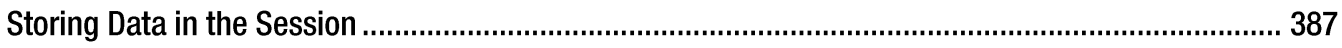

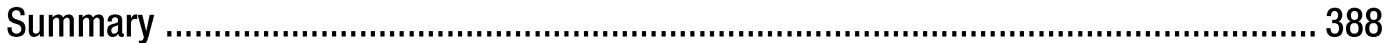

Chapter 18: Using jQuery ..................................................................... 389

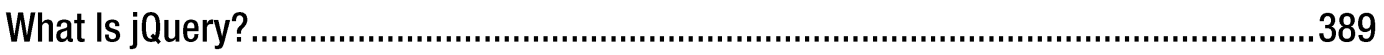

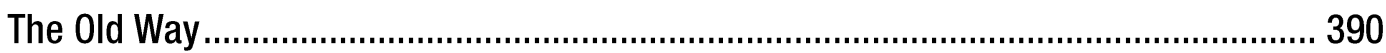




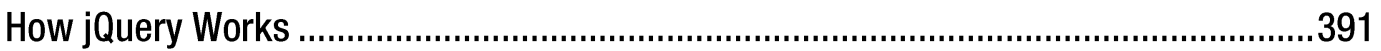

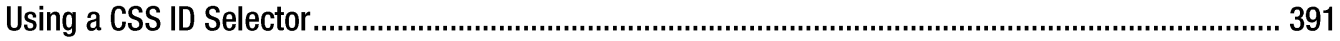

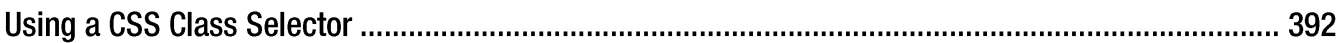

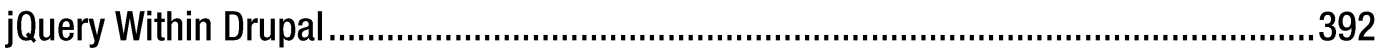

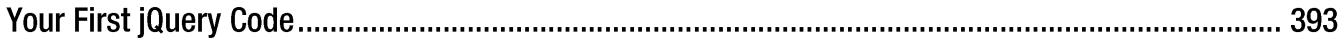

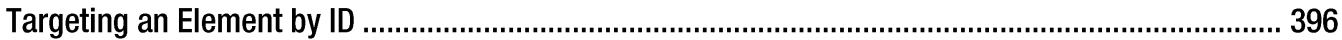

Method Chaining............................................................................................................. 396

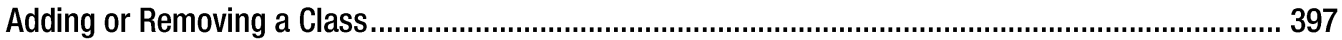

Wrapping Existing Elements ................................................................................................ 397

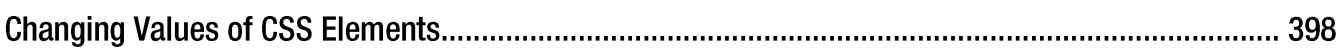

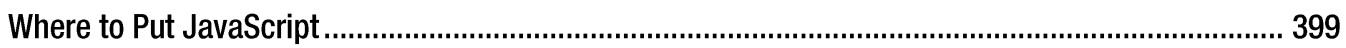

Overridable JavaScript .................................................................................................... 402

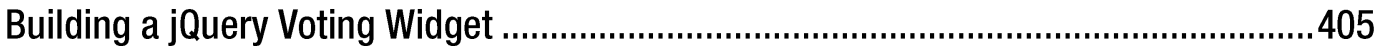

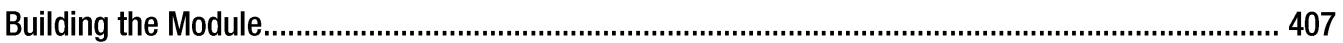

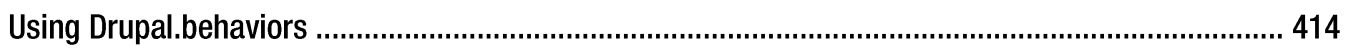

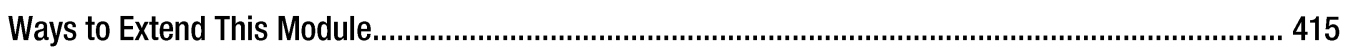

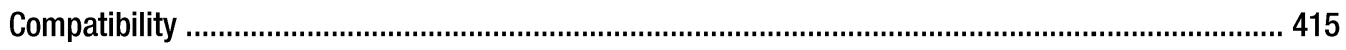

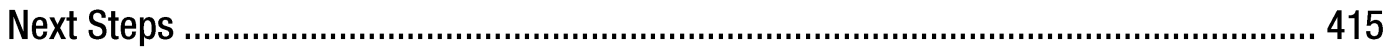

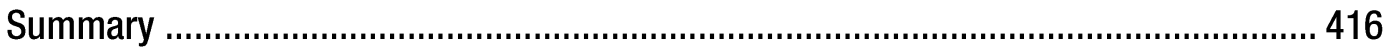

Chapter 19: Localization and Translation .........................................................417

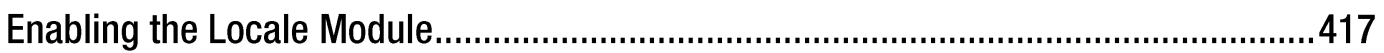

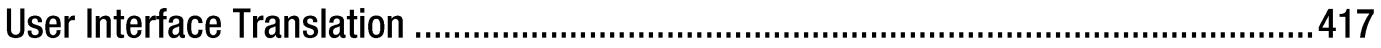

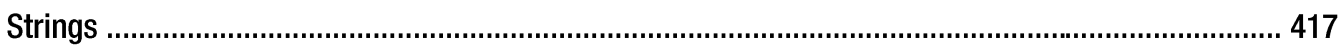

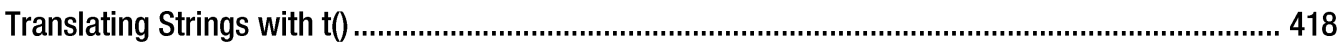

Replacing Built-In Strings with Custom Strings ........................................................................ 419 


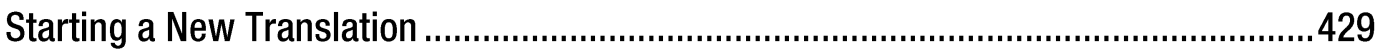

Generating .pot Files with Translation Template Extractor ........................................................... 429

Creating a pot File for Your Module .............................................................................................. 430

Creating .pot Files for an Entire Site...................................................................................... 431

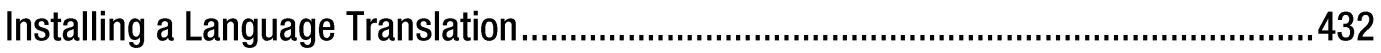

Setting Up a Translation at Install Time...................................................................................... 432

Installing a Translation on an Existing Site.................................................................................. 433

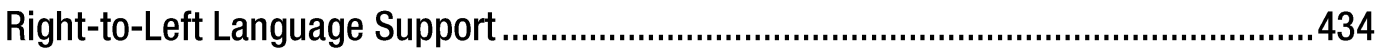

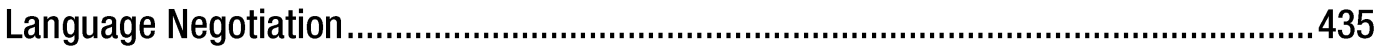

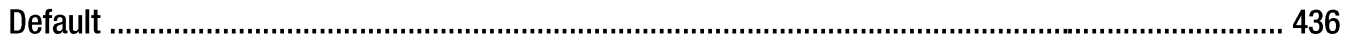

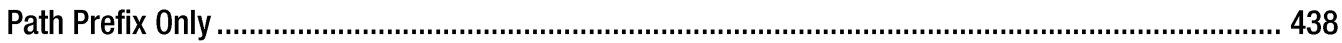

Path Prefix with Language Fallback ......................................................................................... 440

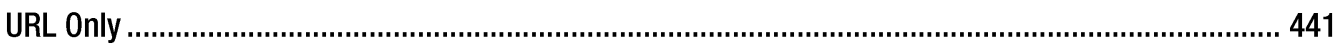

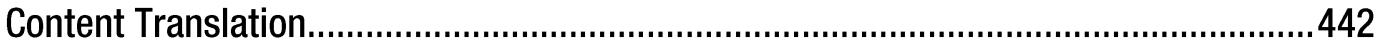

Introducing the Content Translation Module .............................................................................. 442

Multilingual Support .......................................................................................................... 442

Multilingual Support with Translation .................................................................................... 444

Localization- and Translation-Related Files ...........................................................447

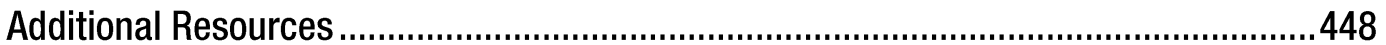

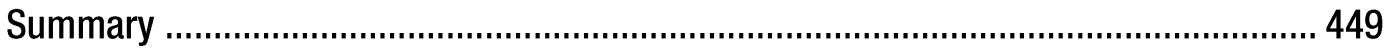

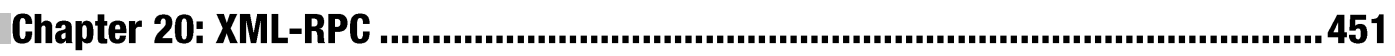

What Is XML-RPC?

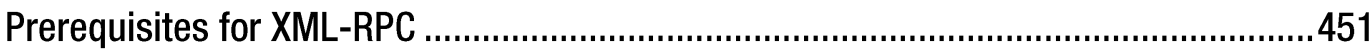

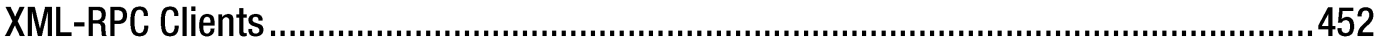

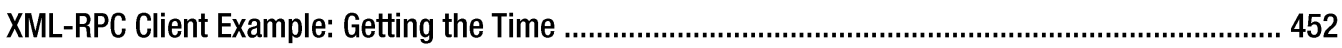

XML-RPC Client Example: Getting the Name of a State.............................................................. 453

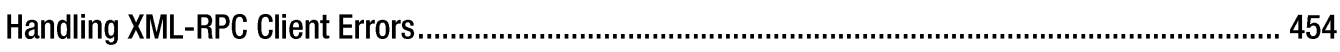




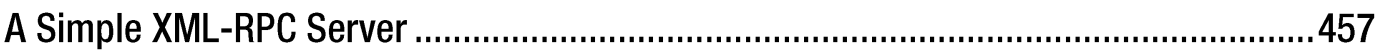

Mapping Your Method with hook_xmlrpc) ………................................................................ 458

Automatic Parameter Type Validation with hook_xmlrpc() …….................................................... 459

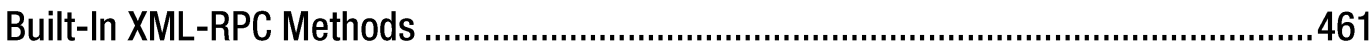

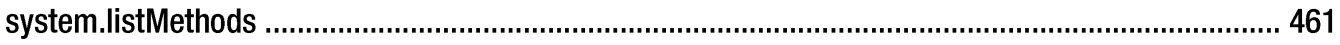

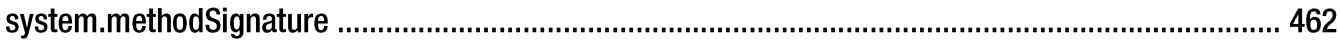

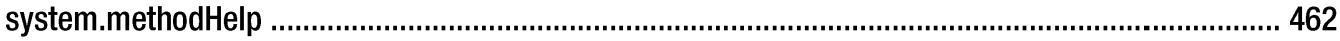

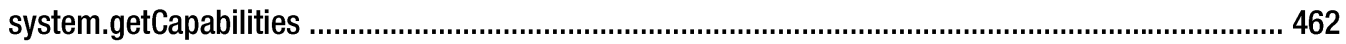

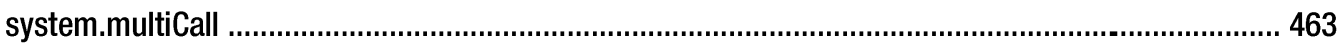

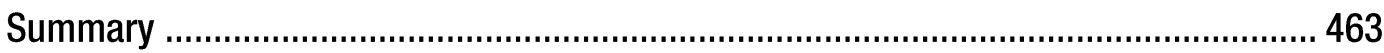

Chapter 21: Writing Secure Code...................................................................... 465

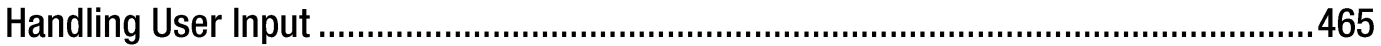

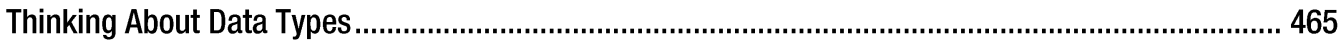

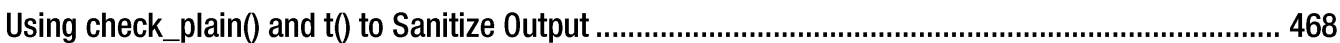

Using filter_xss) to Prevent Cross-Site Scripting Attacks .............................................................. 470

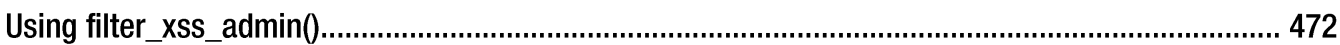

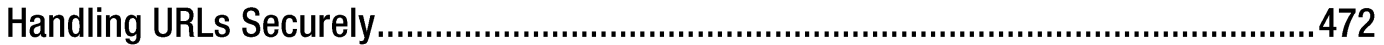

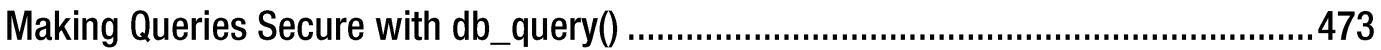

Keeping Private Data Private with hook_query_alter() .............................................476

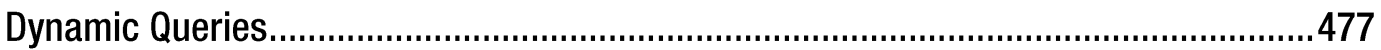

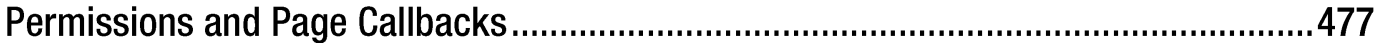

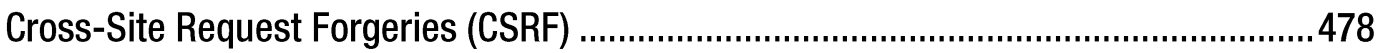

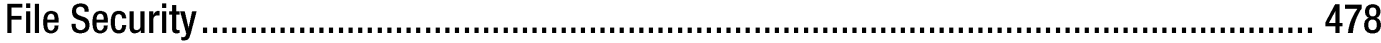

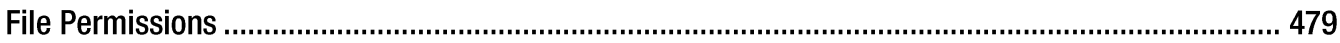

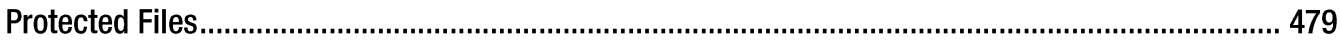

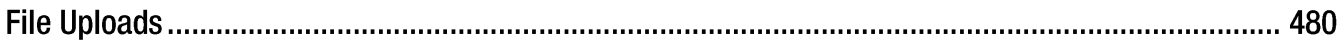

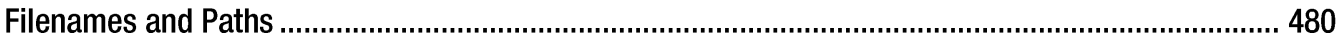

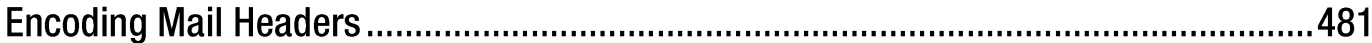


Files for Production Environments......................................................................... 482

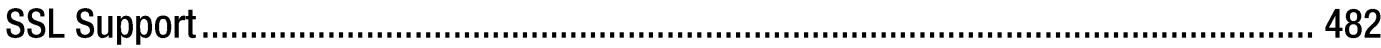

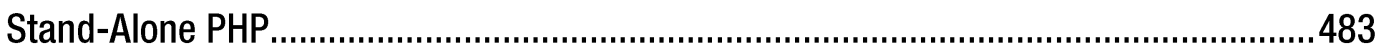

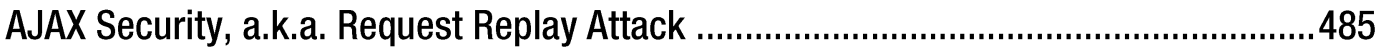

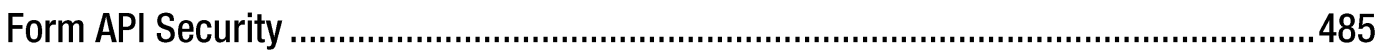

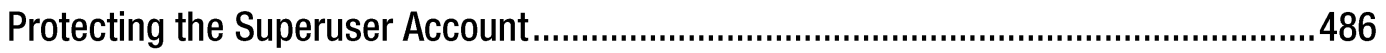

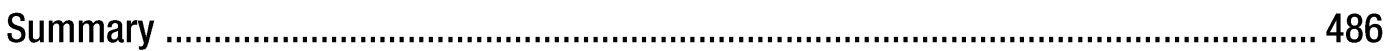

Chapter 22: Development Best Practices .......................................................... 487

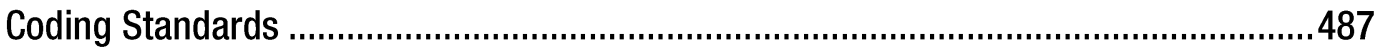

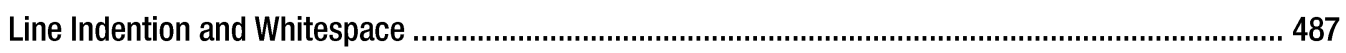

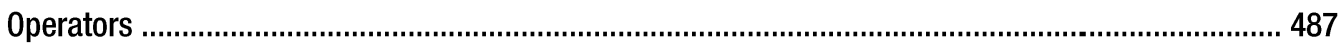

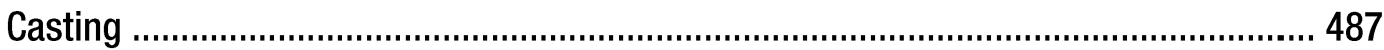

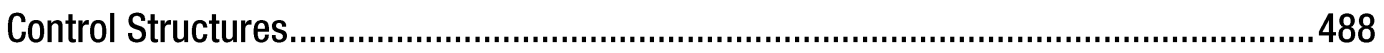

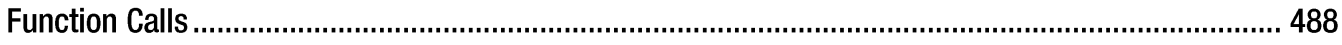

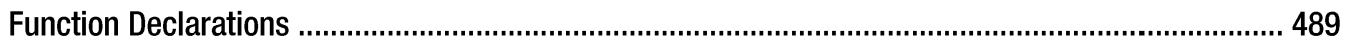

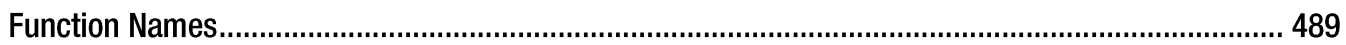

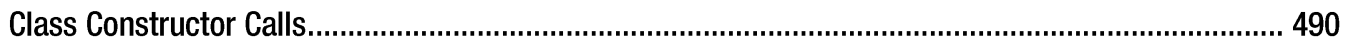

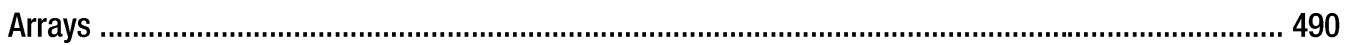

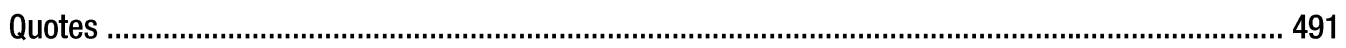

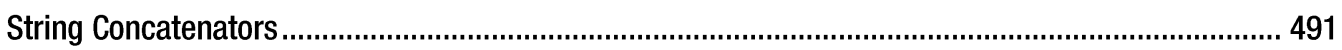

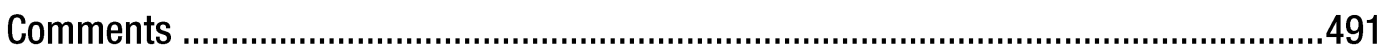

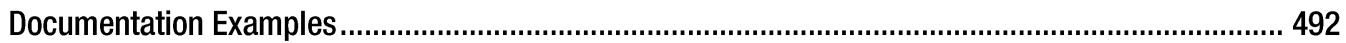

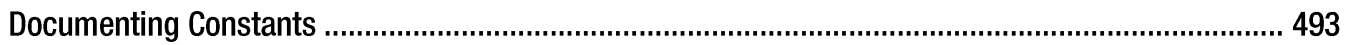

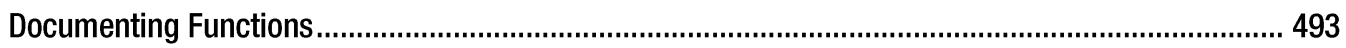

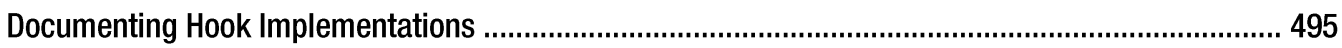

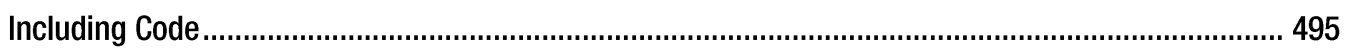

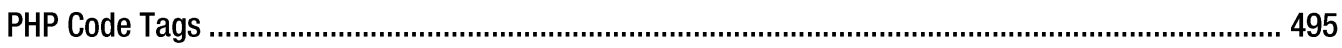

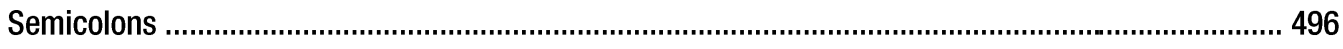


Example URLs. 496

Naming Conventions...........................................................................................496

Checking Your Coding Style with Coder Module....................................................496

Finding Your Way Around Code with grep ..............................................................497

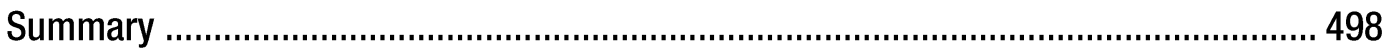

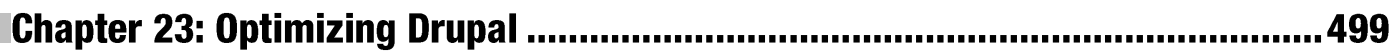

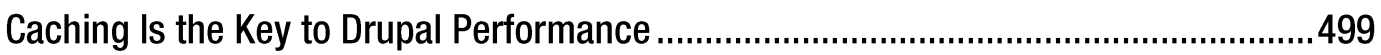

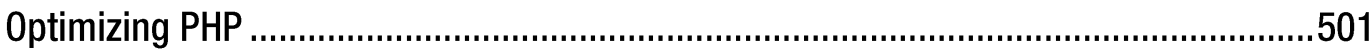

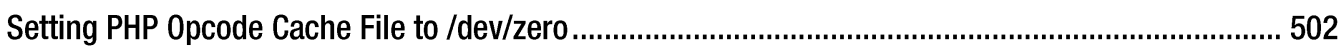

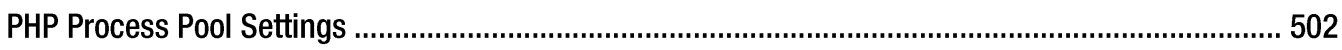

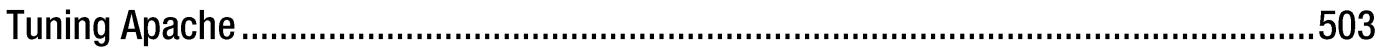

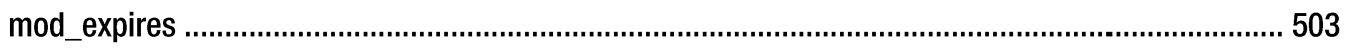

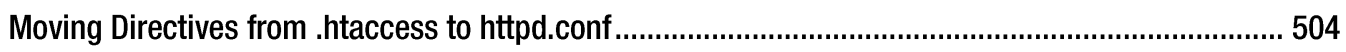

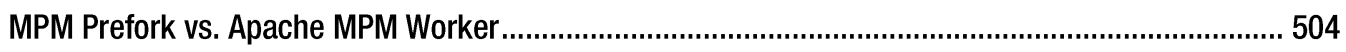

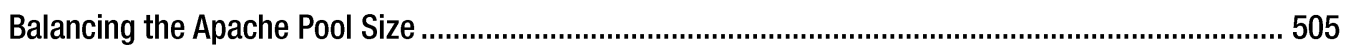

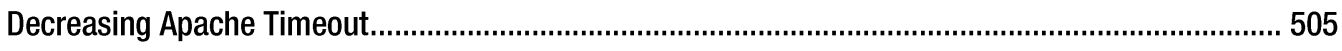

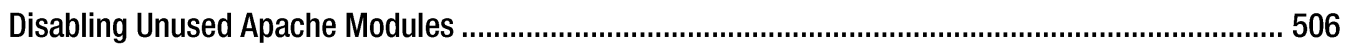

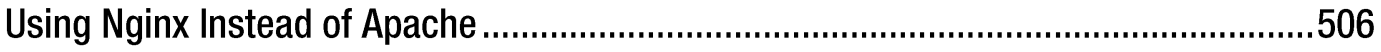

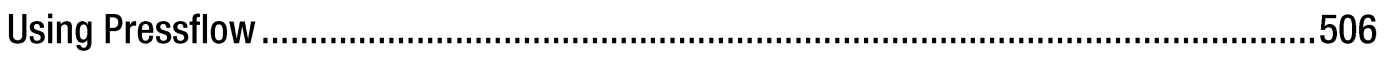

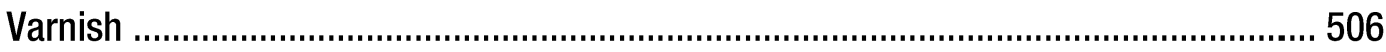

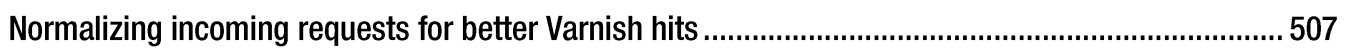

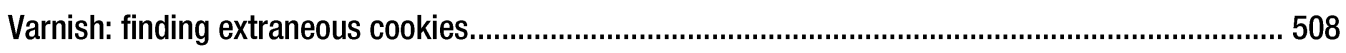

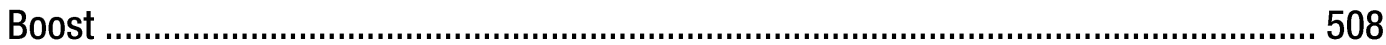

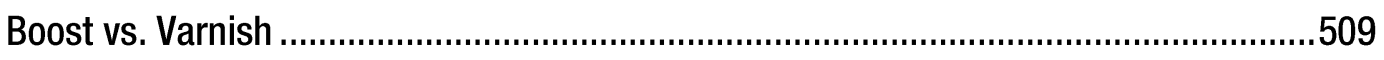


Linux System Tuning for High Traffic Servers .......................................................509

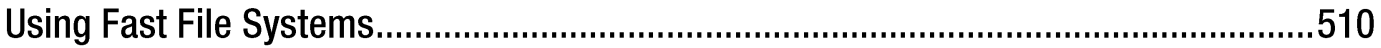

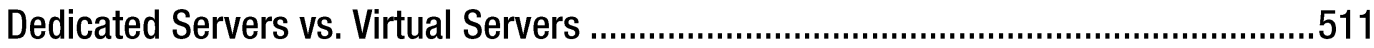

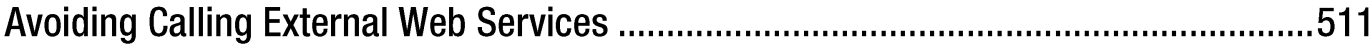

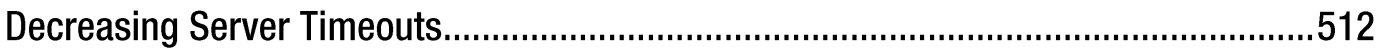

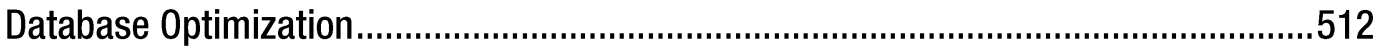

Enabling MySQL's Query Cache.......................................................................................... 512

MySQL InnoDB Performance on Windows .............................................................................. 513

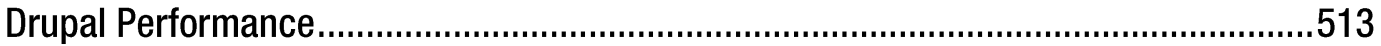

Eliminating 404 Errors.................................................................................................... 513

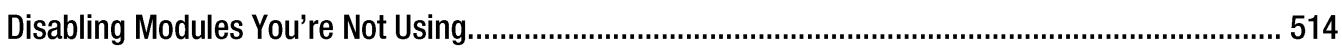

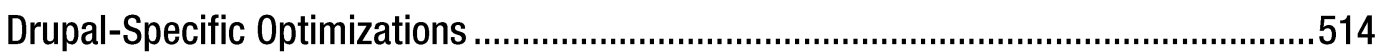

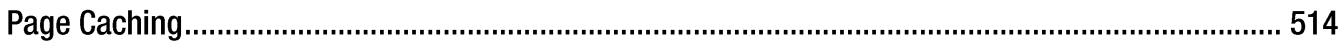

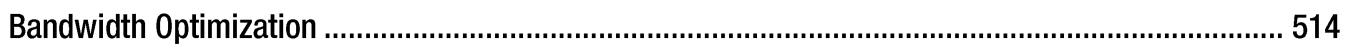

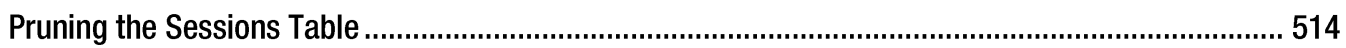

Managing the Traffic of Authenticated Users ...................................................................... 515

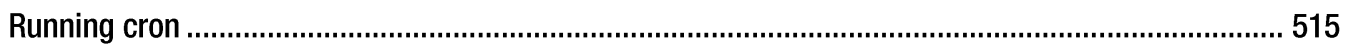

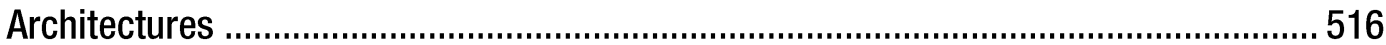

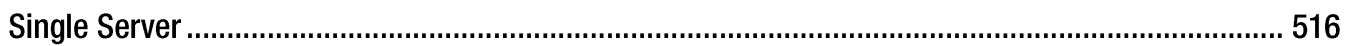

Separate Database Server .............................................................................................. 516

Separate Database Server and a Web Server Cluster …………….............................................. 517

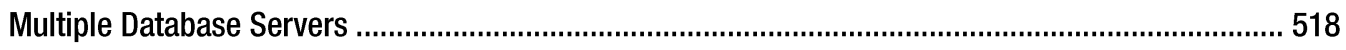

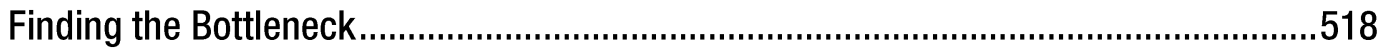

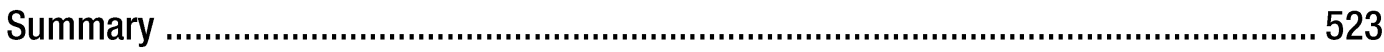


Chapter 24: Installation Profiles 525

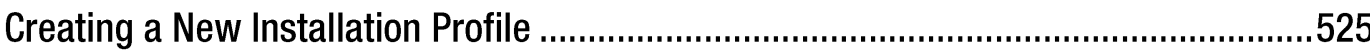

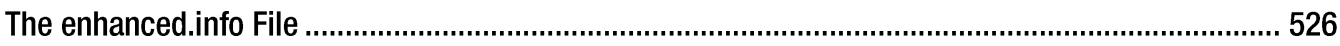

The enhanced.profile File ................................................................................................... 527

The enhanced.install File........................................................................................................... 527

Using hook_install_tasks and hook_install_tasks_alter ...........................................543

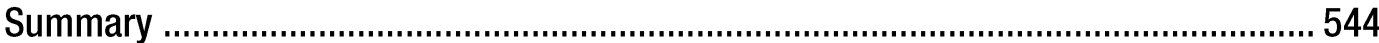

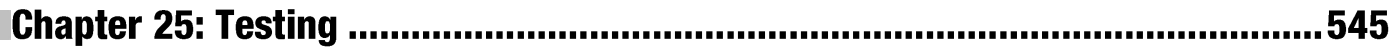

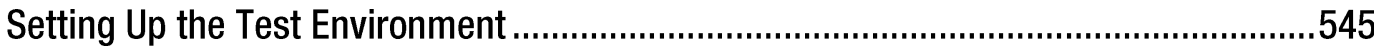

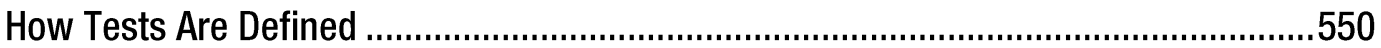

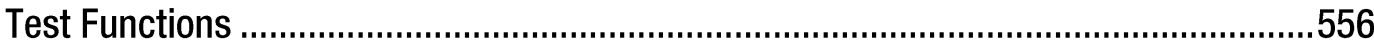

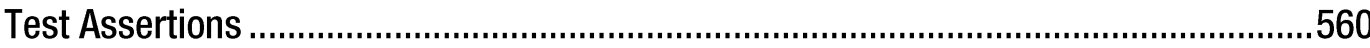

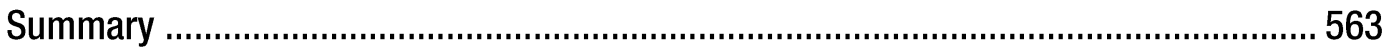

Appendix A: Database Table Reference .........................................................565

Appendix B: Resources.......................................................................................623

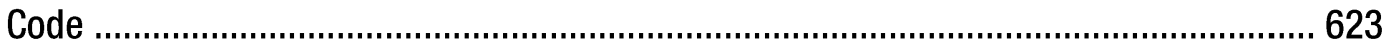

The Drupal Source Code Repository on GIT ............................................................................ 623

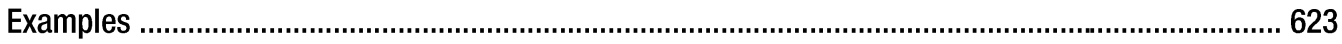

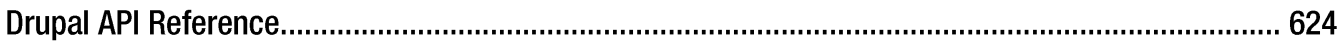

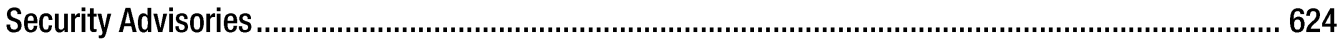

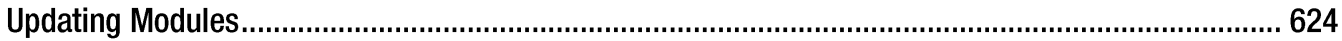

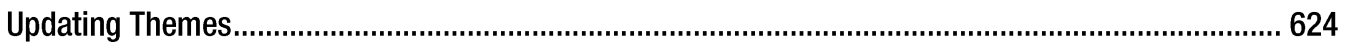

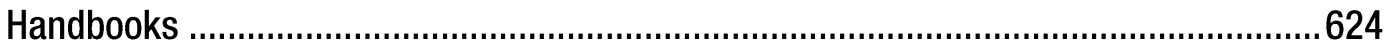

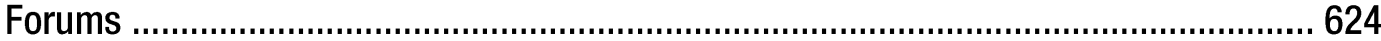


Mailing Lists

Index 


\section{Foreword}

Four years ago, I wrote the foreword for the first edition of this book. What was missing at that time was a developer book for Drupal. Since then, Pro Drupal Development has made an incredible contribution to Drupal's steady growth. I don't think I know a single Drupal developer who doesn't own a copy of the Pro Drupal Development book.

Drupal, through its open source nature, has become much greater than I ever imagined it would. What didn't change is the Drupal developer community's healthy desire to innovate, to respond to the ever-changing landscape of web development, and to provide web developers an almost infinite amount of flexibility. Change is a constant in the Drupal community and key to our success.

A lot of the success of Drupal today can be attributed to Drupal 6. However, from the day that Drupal 6 was released almost three years ago, we've been working really hard on Drupal 7. More than 800 individual contributors have patches included in Drupal 7 core. Drupal 7 will feature some of the biggest architectural changes in the history of Drupal, will ship with many API improvements, and will be able to power bigger sites than ever before. The net result is that Drupal 7 is an even better web application development platform than Drupal 6, and it will fuel a lot of Drupal's growth over the next years.

All these changes also mean that the previous Pro Drupal Development books went out of date. Fortunately, the third edition of this book fixes all that. This book covers all of the capabilities and developer facilities in Drupal 7, and provides deep insight into the inner workings and design choices behind Drupal 7.

Armed with this book and a copy of Drupal's source code, you have everything you need to become a Drupal expert. If, along the way, you have figured out how to do something better, with fewer lines of code or more elegantly and faster than before, get involved and help us make Drupal even better. I'd love to review and commit your Drupal core patches, and I'm sure many of the other contributors would too.

Dries Buytaert

Drupal Founder and Project Lead 


\section{About the Authors}

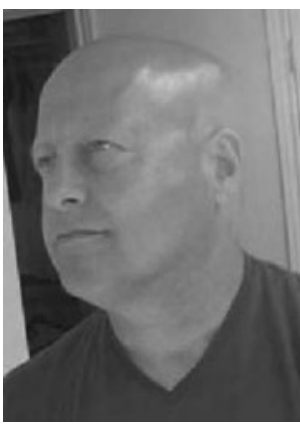

Todd Tomlinson is the vice president of eGovernment Solutions at ServerLogic Corporation in Portland, Oregon. Todd's focus over the past 15 years has been on designing, developing, deploying, and supporting complex web solutions for public and private sector clients all around the world. He has been using Drupal as the primary platform for creating beautiful and featurerich sites such as http://arapahoelibraries.org/ald/.

Prior to ServerLogic, Todd was the senior director of eBusiness Strategic Services for Oracle Corporation, where he helped Oracle's largest clients develop their strategic plans for leveraging the Web as a core component of their business. He is also the former vice president of Internet Solutions for Claremont Technology Group, vice president and CTO of Emerald Solutions, managing director for CNF Ventures, and a senior manager with Andersen Consulting/Accenture. Todd has a BS in computer science and an MBA, and he is in the dissertation phase for his $\mathrm{PhD}$.

Todd's passion for Drupal is evident in his obsession with evangelizing about the platform and his enthusiasm when speaking with clients about the possibilities of what they can accomplish using Drupal. If you want to see someone literally "light up," stop him on the street and ask him, "What is Drupal and what can it do for me?" He is also the author of Apress's Beginning Drupal 7.

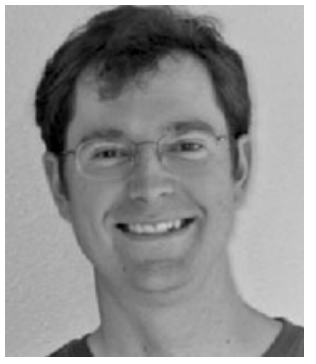

John K. VanDyk began his work with computers on a black Bell and Howell Apple II by printing out and poring over the BASIC code for Little Brick Out in order to increase the paddle width. Later, he manipulated timing loops in assembly to give Pac-Man a larger time slice than the ghosts. Before discovering Drupal, John was involved with the UserLand Frontier community and used Plone before writing his own content management system (with Matt Westgate) using Ruby.

John is a senior web architect at Lullabot, a Drupal education and consulting firm. Before that, John was a systems analyst and adjunct assistant professor in the entomology department at Iowa State University of Science and Technology. His master's thesis focused on cold tolerance of deer ticks, and his doctoral dissertation was on the effectiveness of photographically created three-dimensional virtual insects on undergraduate learning.

John lives with his wife Tina in Ames, Iowa. They homeschool their passel of children, who have become used to bedtime stories like "The Adventures of a Node Revision in the Land of Multiple Joins." 


\section{About the Technical Reviewers}

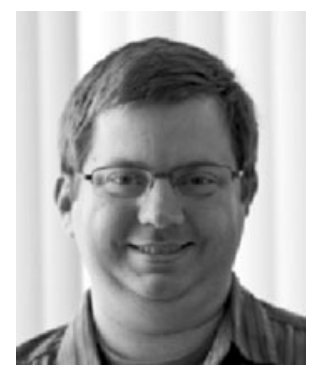

Joshua Brauer jumped onto the World Wide Web as an aspiring technical journalism student working with content management systems in 1995. Since becoming a member of the Drupal community in 2003, Joshua has been involved with running Drupal sites of all sizes. In 2007 Joshua left work in IT management to devote his full-time professional effort to Drupal.

Joshua is one of the leaders of the Boise Drupal Users Group and can frequently be found giving talks at conferences, camps, local meetups, and anywhere else people are interested in hearing about Drupal. Joshua's writings about Drupal can be found online at http://joshuabrauer.com.

As a Drupalist at Acquia, Joshua works with customers from small sites to large enterprises on all phases of their Drupal experience, from pre-planning through hosting and operations. Joshua finds great inspiration in the Drupal community and its many significant accomplishments. When disconnected from the Web, Joshua can be found behind a camera, enjoying the wonderful variety of beautiful places on our planet.
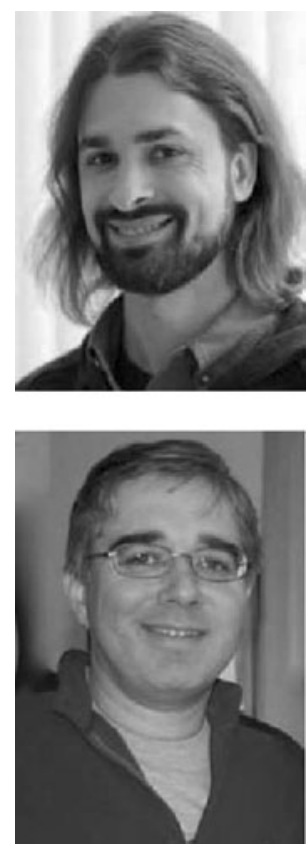

Robert Douglass is the senior Drupal advisor at Acquia, Inc., a permanent member of the Drupal Association, and a founding member of Die DrupalInitiative, Germany's Drupal-oriented nonprofit. He is active as a module maintainer, core contributor, and speaker at various Drupal events and conferences. His Apress projects include Building Online Communities with Drupal, phpBB, and WordPress (author, 2005), Pro Drupal Development (technical reviewer, 2007), and Pro Drupal Development, Second Edition (technical reviewer, 2008).

Peter M. Wolanin has been working with Drupal since late 2005, when a friend who had been a Howard Dean supporter involved him in a project to build a new Web presence for the local Democratic Party club, and they started building the site on Drupal 4.7 beta. Peter soon became as interested in the challenge of fixing bugs and adding features in Drupal core and contributed modules as he was in actual site building. He became a noted contributor to Drupal 5, 6, and 7, and a member of the Drupal documentation team. He joined the Drupal security team and was elected in 2010 as a permanent member of the Drupal Association. Peter joined the Acquia engineering team in 2008 and enjoys the company of his stellar colleagues. Before all this, Peter graduated cum laude from Princeton University, received a doctoral degree in physics from the University of Michigan, and conducted post-doctoral and industrial research in biophysics and molecular biology. 


\section{Acknowledgments}

Beth, for your never-ending support, encouragement, love, and laughter-thank you for bringing back the ability to dream big about the future.

My daughters, Anna, Alissa, and Emma, for giving up countless hours of time with Dad while I wrote the book.

My parents, for giving me the tools I needed to embark on the journeys that I've traveled.

My grandmother, for sparking the fire to become an author.

Dries, without your vision and passion for the platform, there wouldn't be a Pro Drupal Development book.

The Aquia team, for jumping in and lending your support while I tackled the tough sections of the book Webchick (a.k.a. Angie Byron), for your dedication to the platform and your relentless efforts to launch Drupal 7.

The thousands of developers who have contributed to the platform to make it what it is today.

My clients, for embracing the technology and sharing the excitement over what it can do.

Jason, Darren, Kathryn, and Steve-my teammates who wake up every morning excited to discover something new that Drupal can do-for putting up with my wild dreams about how Drupal can do anything. 


\section{Introduction}

In its relatively short life, Drupal has had a tremendous impact on the landscape of the Internet. As a web content management system, Drupal has enabled the creation of feature- and content-rich web sites for organizations large and small. As a web application framework, Drupal is changing the way that people think about web application development. When I experienced the power of the Drupal platform for the first time, I knew that it was something more than just another content management solution. When I saw how easily and quickly I could build feature-rich web sites, I shifted gears and focused my entire career around Drupal.

I'm often asked the question, "What is Drupal?" The short answer is Drupal is an open source web content management system that allows you to quickly and easily create simple to complex web sites that span everything from a simple blog to a corporate web site, a social networking web site, or virtually anything you can dream up. What you can build with Drupal is limited only to your imagination, the time you have to spend with the platform, and your knowledge about Drupal's capabilities - which is the impetus behind this book.

As an open source platform, Drupal's community is constantly improving the platform and extending the functionality of the core platform by creating new and exciting add-on modules. If there's a new concept created on the Web, it's likely that there will be a new Drupal module that enables that concept in a matter of days. It's the community behind the platform that makes Drupal what it is today, and what it will become in the future. I'll show you how to leverage the features contributed by the community, making it easy for you to build incredible solutions with minimal effort.

The very act of picking up this book is the first step in your journey down the path of learning how to use Drupal. If you will walk with me through the entire book, you'll have the knowledge and experience to build complex and powerful Drupal-based web sites. You'll also have the foundation necessary to move beyond the basics, expanding on the concepts I cover in this book.

Learning Drupal is like learning every new technology. There will be bumps and hurdles that cause you to step back and scratch your head. I hope the book helps smooth the bumps and provides you with enough information to easily jump over those hurdles. I look forward to seeing your works on the Web and hope to bump into you at an upcoming DrupalCon.

I will end on a note of carefree abandon-learn to steal! Once you've learned the pieces of the puzzle and how to combine them, there is very little new to invent. Every new idea you discover is a mere permutation of the old ideas. And ideas are free! Every cool feature discussed on TV shows or presented in the brochures or web sites of commercial HA companies can be taken, adapted, and implemented with the information presented here using very little effort. And then you will graduate from an automated home to a smart home to a personalized smart home! 